\title{
On Pulse Position Modulation and Its Application to PLLs for Spur Reduction
}

\author{
Chembiyan Thambidurai and Nagendra Krishnapura
}

\begin{abstract}
Randomizing the positions of charge pump current pulses in a PLL breaks their periodicity and redistributes the reference spurs into broadband noise. Closed form expressions for the power spectral density (PSD) of pulse position modulated (PPM) signals are derived and intuitive explanations for the results are given. The redistributed noise has a high-pass shape and does not affect the close in phase noise of the PLL. PPM using a uniformly distributed i.i.d. sequence completely removes the spurs and provides a first-order shaping of redistributed noise. Higher order shaping and reduction of redistributed noise at intermediate offset frequencies are possible using PPM with a high-pass shaped modulating sequence and pulse repetition. Circuit implementations of these techniques are given and their nonidealities are discussed. Simulation results from a $1 \mathrm{GHz}$ PLL operating from a reference frequency of $20 \mathrm{MHz}$ and a bandwidth of $1 \mathrm{MHz}$ confirm the results of the analysis and viability of the proposed techniques. In the presence of nonidealities spurs can be reduced by at least $13 \mathrm{~dB}$ without any trimming of the delays in the PPM circuits and by $25 \mathrm{~dB}$ after trimming the delays to within $5 \%$ of the nominal value.
\end{abstract}

Index Terms-Digital modulation, phase locked loops (PLL), phase noise, random processes, signal analysis, spectral analysis.

\section{Motivation}

I $\mathrm{N}$ THE STEADY state of a charge pump PLL, the divide and reference edges align and the charge pump current should ideally be zero. In reality, due to circuit nonidealities like charge pump current mismatch, loop filter leakage, and feedthrough of the charge pump switches, a nonzero current $i_{\mathrm{cp}}(t)$ which is periodic at the reference frequency $\left(f_{r}=1 / T\right)$ is injected into the loop filter as shown in Fig. 1. This generates a periodic disturbance on the control voltage and manifests itself as a reference spur at the PLL output. The additional VCO output phase noise spectrum due to the charge pump noise can be expressed in $\mathrm{dBc}$ as [1]

$$
S_{\phi}(f)=10 \log \left(S_{\mathrm{cp}}(f)\left|\frac{Z(f) K_{\mathrm{vco}}}{f}\right|^{2}\right)
$$

where $S_{\mathrm{cp}}(f)$ is the PSD of $i_{\mathrm{cp}}(t)$. When $i_{\mathrm{cp}}(t)$ is periodic at $f_{r}, S_{\mathrm{cp}}(f)$ (and hence $S_{\phi}(f)$ ) consists of impulses, or spurs, at integer multiples of $f_{r}$. To reduce spurs, the product $Z(f) K_{\mathrm{vco}}$

Manuscript received October 09, 2010; revised January 23, 2011; accepted April 11, 2011. Date of publication June 16, 2011; date of current version June 29, 2011. This work was supported in part by the Ministry of Information Technology, Government of India. This paper was recommended by Editor G. Manganaro.

The authors are with the Department of Electrical Engineering Indian Institute of Technology—Madras, Chennai, 600 036, India (e-mail: chembiant@gmail. com).

Digital Object Identifier 10.1109/TCSI.2011.2157749

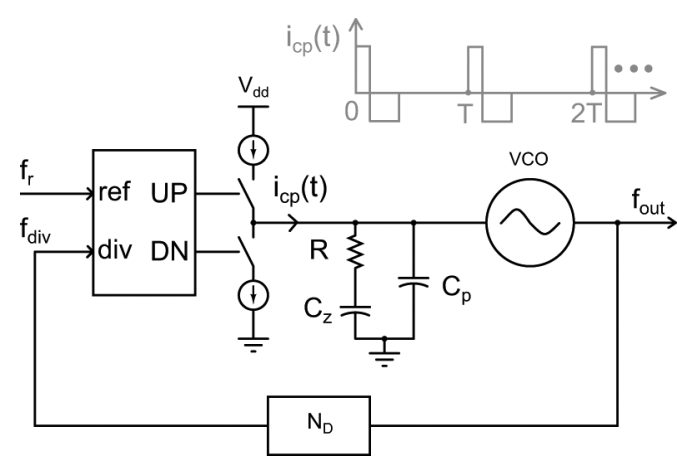

Fig. 1. The standard charge pump PLL architecture showing the periodic charge pump current in steady state.

has to be reduced. This implies a proportionally smaller bandwidth for a given loop stability margin. Several techniques [2]-[6] have been proposed to address this problem of spur magnitude and bandwidth trade-off. Reference [2] addresses the issue based on the technique of delay-sampling the control voltage. But this technique is not effective in the presence of loop filter capacitor leakage. Reference [3] minimizes the charge pump mismatch thereby reducing the spur, at the cost of increased settling time. Reference [4] uses distributed charge pump and phase frequency detector (PFD) with pulse position randomization to reduce the spur. Using distributed PFD and charge pumps can cause the total size of the charge pump switches to be larger, increasing the net feedthrough error besides an increase in implementation complexity.

Random positioning of pulses of the charge pump current $i_{\mathrm{cp}}(t)$ within the reference period $T$ [4], [5], in other words, pulse position modulation (PPM) by a random sequence, breaks the periodicity and distributes the energy in the reference spurs into wideband phase noise. In order to quantify this effect, the spectral density of random PPM signal $\left[S_{\mathrm{cp}}(f)\right.$ in (1)] has to be determined. In this work, we present a general analysis of pulse trains whose amplitude and pulse positions are modulated by independent stationary sequences with arbitrary probability distributions and give closed form expressions for the PSD. This analysis is simpler and more intuitively understood compared to previously published results [7]. We also analyze the effects of pulse repetition (PR) within a reference period [4], and the combination of PPM and PR. These analyses enable us to determine the phase noise due to redistributed spur energy, and also to derive schemes to reduce the output phase noise in the intermediate frequency range of $f_{r} / 10$ to $f_{r} / 100$ by exploiting correlations in the modulating sequence.

The proposed spur reduction techniques involve delaying UP and DN signals which control $i_{\mathrm{cp}}(t)$ by random amounts, which 
can be accomplished using a digital delay line and a MUX controlled by a random sequence [5]. A similar approach also results in a simpler implementation of PR compared to [4]. The proposed implementation is less sensitive to delay line mismatch than when the delay lines are placed before the PFD [4]. Unlike the latter, it requires delaying narrow pulses with a minimum width equal to the reset delay of the PFD. Design trade-offs due to these constraints are analyzed. Modifications to the implementation are suggested for cases when the reset delay is very small.

The mathematical formulation of the problem and analytical expressions are presented in Section II. Section III discusses the application of PPM to address the problem of reference spurs in a charge pump PLL and presents a detailed study of PPM by a uniformly distributed sequence. Section IV discusses the different PPM techniques for spur reduction with their implementation details. Section $\mathrm{V}$ addresses the details of the delay line design and alternative implementation methods. Section VI discusses the details of modulating sequence generation. Section VII discusses the simulation results. A detailed analysis of the effect of jitter and random mismatch of the delay line is presented in Section VIII. Section IX presents the conclusions drawn from the work. Appendix I gives the detailed derivation of the PSD of the PPM signals.

\section{MATHEMATICAL FORMULATION}

Let $x_{k}$ and $a_{k}$ be two stationary sequences. Then

$$
x_{p}(t)=\sum_{k=-\infty}^{\infty} x_{k} \delta(t-k T)
$$

is a pulse amplitude modulated (PAM) signal [8] of period $T$.

$$
r_{p}(t)=\sum_{k=-\infty}^{\infty} x_{k} \delta\left(t-k T-a_{k} T_{d}\right)
$$

is a pulse position and amplitude modulated (PPAM) signal whose pulse positions $a_{k} T_{d}$ are modulated by the sequence $a_{k} \in$ $[0, N-1]$ and pulse amplitudes are modulated by $x_{k}$. The pulse shape $p(t)$ is assumed to be an impulse for simplicity of expressions. Fig. 2 illustrates PAM and PPAM signals for $N=8$ and $T_{d}=T / N$. The signals $x_{p}(t)$ and $r_{p}(t)$ are cyclostationary random processes [9], when the modulating sequences $x_{k}$ and $a_{k}$ are stationary. The power spectral density (PSD) $S_{x p}(f)$ of the PAM signal is given by [8]

$$
S_{x p}(f)=\frac{1}{T} S_{x}(f)
$$

$S_{x}(f)$ is the PSD of the stationary sequence $x_{k}$ (with an autocorrelation function $R_{x}(k)$ ) given by $S_{x}(f)=$ $\sum_{k} R_{x}(k) e^{-j 2 \pi f k T}$. If the samples of the sequence $a_{k}$ are i.i.d., the PSD $S_{r p}(f)$ of PPAM signal is given by (Appendix I)

$$
S_{r p}(f)=\underbrace{\frac{1}{T} S_{x}(f)|C(f)|^{2}}_{\text {Power filtered }}+\underbrace{\frac{R_{x}(0)}{T}\left[1-|C(f)|^{2}\right]}_{\text {Power redistributed }}
$$

where

$$
C(f)=\sum_{m=0}^{N-1} P_{A}(a=m) e^{-j 2 \pi f m T_{d}} .
$$

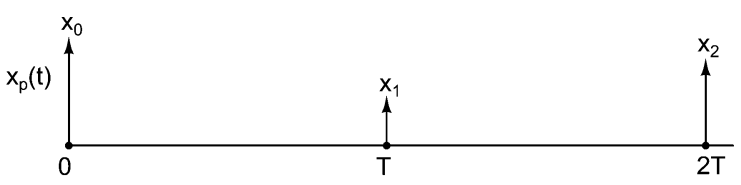

(a)

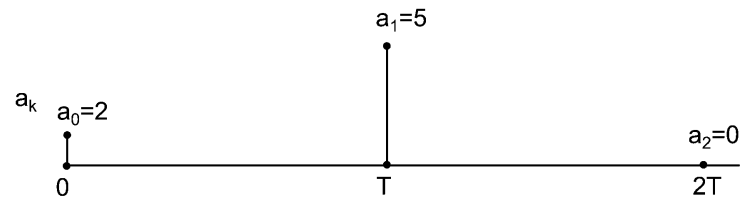

(b)

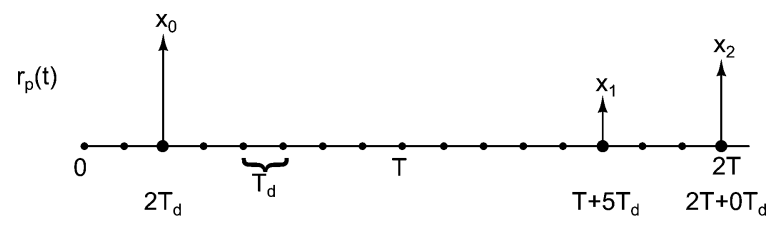

(c)

Fig. 2. (a) PAM with sequence $x_{k}$ (b) Pulse position modulating sequence $a_{k}$ (c) PPAM with sequences $x_{k}$ and $a_{k}$ for $N=8$.

$P_{A}(a)$ is the probability mass function of the sequence $a_{k} . C(f)$ is what we call the "pseudofilter." It is the characteristic function [8] of the modulating sequence's probability mass function. For any arbitrary pulse shape $p(t)$ the PSD of the PAM and PPAM signals is obtained by multiplying the above expressions by $|P(f)|^{2}$, where $P(f)$ is the Fourier transform of the pulse $p(t)$. When $p(t)$ represents the charge pump current, the pulses are much narrower than the reference period $T$ and have an average value of zero.

Equation (5) shows that the PPAM is equivalent to passing the PAM signal through a filter $C(f)$ and the power that is filtered out (lost) is redistributed as a continuous wideband noise. The characteristics of the filter and the noise are completely determined by the probability distribution of the randomizing sequence $a_{k}$. Since $P_{A}(a=m)$ is positive for all $m$ and $\sum_{m} P_{A}(a=m)=1, C(f)$ is a low-pass filter with unity dc gain and the redistributed power has a high-pass shape.

One can intuitively see that, on an average over a large number of clock cycles, the samples of $x_{p}(t)$ would have occupied the positions $m T_{d}$ within the single interval $T$, with weights $P_{A}(a=m)$. A scaled summation of the delayed versions of the signal $x_{p}(t)$ is nothing but low-pass filtering of the signal.

\section{PPM With UNIFORMLY DistRIBUTED I.I.D. SEQUENCE}

Let $x_{i}(t)$ be a periodic impulse train of period $T, x_{i}(t)=$ $\sum_{k} \delta(t-k T) . x_{i}(t)$ is a PAM signal with $x_{k}$ always equal to 1, hence $R_{x}(k)=1$. Using (4), the PSD $S_{x i}(f)$ of $x_{i}(t)$ is given by

$$
S_{x i}(f)=\frac{1}{T} \sum_{k} e^{-j 2 \pi f k T}=\frac{1}{T^{2}} \sum_{k} \delta\left(f-k f_{r}\right) .
$$

By virtue of its periodicity, the power of the signal is concentrated only at the harmonics of the fundamental frequency $\left(f_{r}=\right.$ $1 / T)$. Let $a_{k}$ be of uniform distribution $\in[0, N-1]$ and $T_{d}=$

\footnotetext{
${ }^{1}$ Since it is not an actual filter.
} 
$T / N$. Using (5), the PSD $S_{r}(f)$ of the resulting $N$-PPM ( $N$ represents the number of pulse positions of the PPM signal) signal $r(t)$ can be computed to be

$S_{r}(f)=\frac{1}{T^{2}} \sum_{k=-\infty}^{\infty} \delta\left(f-k f_{r}\right)\left|C_{N}(f)\right|^{2}+\frac{1}{T}\left[1-\left|C_{N}(f)\right|^{2}\right]$.

Since $P\left(a_{k}\right)=1 / N$, the squared magnitude of the associated pseudofilter $C_{N}(f)$ from (6) is given by

$$
\left|C_{N}(f)\right|^{2}=\left|\frac{1}{N} \sum_{l=0}^{N-1} e^{-j 2 \pi f l T / N}\right|^{2}=\left(\frac{\sin (\pi f T)}{N \sin \left(\frac{\pi f T}{N}\right)}\right)^{2} .
$$

$C_{N}(f)$ is a moving average filter of length $N$ with nulls at $k f_{r}$ where $k \in[1, N-1]$. Thus, the spectrum of $N$-PPM reduces to

$$
S_{r}(f)=\frac{1}{T^{2}} \sum_{k=-\infty}^{\infty} \delta\left(f-k N f_{r}\right)+\frac{1}{T}\left[1-\left|C_{N}(f)\right|^{2}\right] .
$$

Comparing (10) to (7) we can see that the $N$-PPM signal contains spurs at the harmonics of $N f_{r}$ and the harmonics in $\left[f_{r},(N-1) f_{r}\right]$ are absent. The second term in (10) is the "redistributed noise" $S_{s n}(f)$

$$
S_{s n}(f)=\frac{1}{T}\left[1-\left|C_{N}(f)\right|^{2}\right] .
$$

It can easily be shown that power lost in the harmonics in $\left[f_{r},(N-1) f_{r}\right]$ is equal to the power in the "redistributed noise" in $\left[0, N f_{r}\right]$. Randomizing the impulse positions by a uniformly distributed sequence $a_{k}$ is equivalent to passing it through a moving average filter. The harmonics in the interval $\left[f_{r},(N-1) f_{r}\right]$ are eliminated and spread as "redistributed noise" $S_{s n}(f)$. Fig. 3 shows the simulated spectrum of the periodic signal and the 8-PPM signal. From the figure we can see that the harmonics in $\left[f_{r}, 7 f_{r}\right]$ are eliminated and spread as noise. The simulated spectral density is coincident with the shape of redistributed noise given by (10). To illustrate the filtering nature of PPM, Fig. 3 also shows the spectrum of the 8-PPM signal overlaid with the associated pseudofilter. We can clearly see the absence of harmonics occurring at the zeroes of the pseudofilter.

$C_{N}(f)$ is a moving average low-pass filter. A moving average filter is a first-order shaped low-pass filter and hence the redistributed noise has a first-order high-pass characteristic. We can easily verify that for $(N=2), S_{s n}(f)=(1 / T) \sin ^{2}(\pi f T / 2)$, which is a first-order high-pass filter. The shape is similar at low frequencies for any $N$. Thus, the PPM technique when applied to a PLL does not affect the close-in phase noise or long term jitter of the PLL.

\section{A. Sensitivity to Delay Variations}

The analysis above revealed that increasing $N$ does not increase the noise shaping at low frequencies but eliminates reference spurs up to $N f_{r}$. But increasing $N$ increases the implementation complexity of the PLL with randomization. Usually eliminating the spurs from the first few harmonics of reference frequency is sufficient as the spurs far away are well rejected by the low-pass characteristic of the PLL loop filter itself. So in
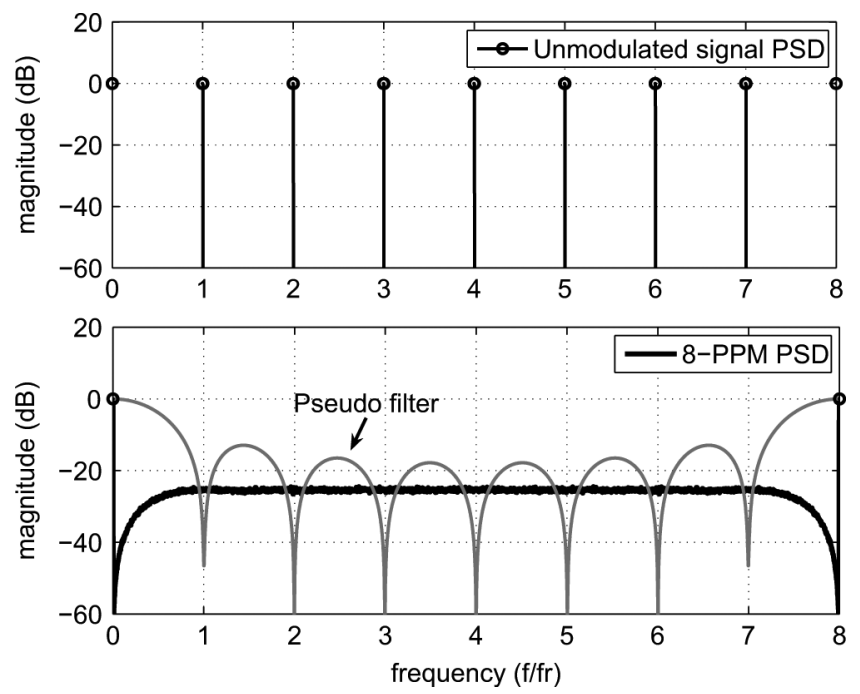

Fig. 3. The spectrum of the unmodulated impulse train and the spectrum of the 8-PPM signal overlaid with the associated pseudofilter (PSD computed with a resolution binwidth of $f_{r} / 512$ ).

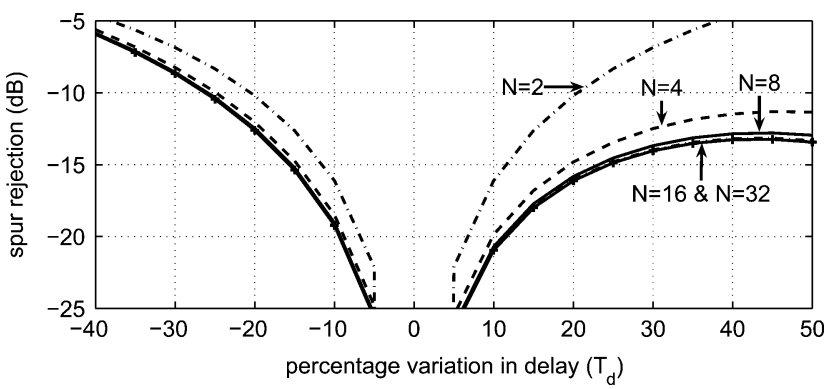

Fig. 4. Sensitivity to delay variations.

order to choose $N$, we consider the sensitivity to delay variations as a measure of performance.

The delays $\left(T_{d}\right)$ are implemented using inverters and are thus prone to systematic variations due to process and temperature. In that case $T_{d} \neq T / N$ and the pseudofilter is given by

$$
\left|C_{N}(f)\right|^{2}=\left(\frac{\sin \left(N \pi f T_{d}\right)}{N \sin \left(\pi f T_{d}\right)}\right)^{2} .
$$

Equation (12) shows that the zeroes of the filter occur at frequencies $f_{z}=k / N T_{d}$, where $k$ is an integer and $k \neq 0, N, 2 N \cdots$. When $T_{d} \neq T / N$, the zeroes of the filter do not occur at multiples of $f_{r}$ and reference spurs appear at the output. Fig. 4 shows the spur rejection when the delay $T_{d}$ varies from the nominal value of $T / N$. As $N$ increases, the sensitivity to delay variations improves up to $N=8$. Beyond that the improvement becomes marginal and therefore we chose $N=8$ for simulations in the implemented PLL.

In the absence of any delay tuning mechanisms, the delays are prone to large variations $( \pm 40 \%)$, hence the spur rejection degrades severely especially for lower values of delays $T_{d}<$ $T / N$ as seen from the figure (6 $\mathrm{dB}$ for $-40 \%$ variation). So to avoid this problem one can choose a skewed nominal delay such that $T_{d} \approx 1.3 T / N$. As seen from the figure, even for $\pm 40 \%$ variations in the skewed delay the spur rejection is at least 13 $\mathrm{dB}$ as opposed to $6 \mathrm{~dB}$ for the nominal delay. Addition of delay trimming mechanisms for process and temperature variations 
i)

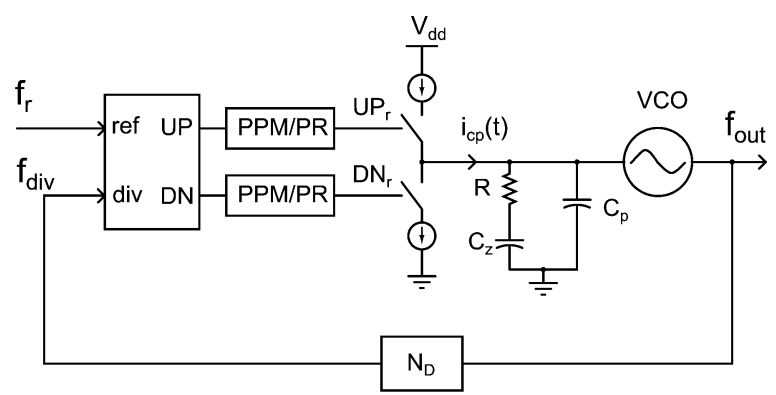

ii)

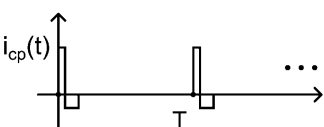

(a)

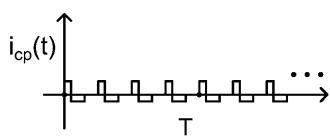

(c)

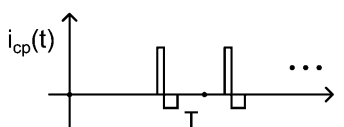

(b)

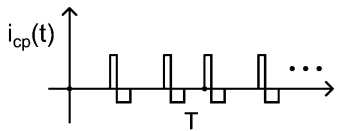

(d)
Fig. 5. (i) The modified PLL architecture with pulse position modulator/pulse repeater shown at block level. (ii) Illustrative waveforms of charge pump current before and after applying the techniques. (a) Standard PLL. (b) PPM. (c) Pulse repetition (PR). (d) PPM+PR

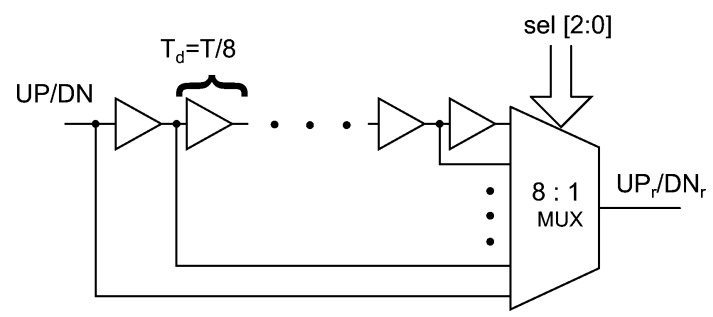

Fig. 6. Implementation of PPM.

can reduce the systematic delay variations to $\pm 5 \%$ resulting in at least $25 \mathrm{~dB}$ spur rejection.

\section{TeChNiQues For SPUR REdUCTION IN A PLL}

Fig. 5 shows the modified PLL architecture employing the PPM based techniques at a block level, with the illustrative charge pump current pulses before and after applying the techniques. The details of the different techniques are described with their pros and cons in the remainder of the section.

\section{A. Random Pulse Position Modulation (PPM)}

As explained in Section III, PPM of charge pump current pulses removes spurs up to $N f_{r}$ and converts them to wideband noise. To implement the technique the pulse position of the UP/DN signals is modulated based on a random control signal sel[2:0]. Modulating the pulse position can be accomplished by delaying the pulses and choosing one of the 8 delayed versions using an 8:1 MUX based on the control signal as shown in Fig. 6.

\section{B. Pulse Repetition (PR)}

Instead of randomizing the pulse positions, we can repeat the pulse $N$ times at intervals of $T / N$ within a single period, making it appear as a high-frequency $\left(N f_{r}\right)$ signal [4]. The charge pump current is reduced by a factor of $N$ to ensure

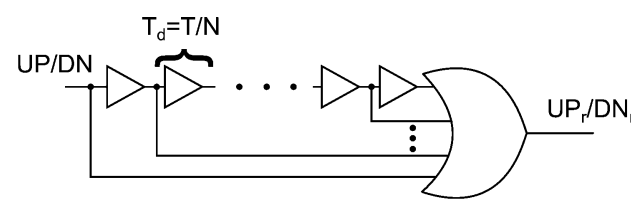

(a)

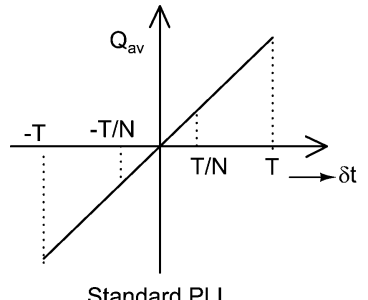

Standard PLL

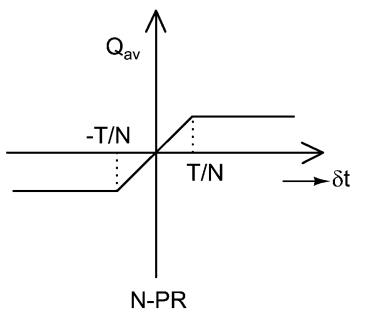

(b)
Fig. 7. (a) Pulse repeater circuit. (b) Charge delivered per reference cycle versus phase error $\delta t$ for the standard PLL and $N$-PR technique.

that the charge delivered by the charge pump per reference cycle remains the same. This is same as passing the current pulse through an $N$-tap moving average filter $C_{N}(f)$. The PSD $S_{p r}(f)$ of the $N$-PR ( $N$ here refers to the number of the repeated pulse positions) signal is

$$
\begin{aligned}
S_{p r}(f) & =\frac{1}{T^{2}}\left|C_{N}(f)\right|^{2} \sum_{k} \delta\left(f-k f_{r}\right) \\
& =\frac{1}{T^{2}} \sum_{k} \delta\left(f-k N f_{r}\right) .
\end{aligned}
$$

In $N$-PR, the harmonics in $\left[f_{r},(N-1) f_{r}\right]$ are filtered out and unlike $N$-PPM there is no additional redistributed noise.

In [4] $N$ scaled charge pumps are driven by delayed UP/DN pulses generated by a distributed PFD. This method of implementation increases the complexity as the number of charge pump cells and PFDs increase with $N$. A simpler implementation would be to drive a single scaled charge pump with a repeated UP/DN pulse, generated by passing $N$ delayed versions of the UP/DN pulses through an $N$-input OR gate as shown in Fig. 7(a). For input phase errors smaller than $T / N$, the proposed implementation of the $N$-PR technique behaves similar to a standard PLL (charge delivered per reference cycle is same for both the cases). For phase errors greater than $T / N$, the delayed UP/DN pulses overlap and the output of the OR-gate is always held high leading to gain saturation (charge delivered remains constant for errors $>T / N$ ) as shown in Fig. 7(b), which increases the settling time of the PLL. In order not to affect the settling behaviour of the PLL, pulse repetition needs to be deactivated and the charge pump current scaled up by a factor $N$ when the PLL is out of lock.

\section{Pulse Position Modulation With Pulse Repetition $(P P M+P R)$}

Since PR removes the reference harmonics without redistributing it as noise, it may seem more attractive than random PPM. However, when the charge pump current is small (of the order of few microamperes), dividing the current further by $N$ may not be possible due to the restrictions imposed by the charge pump mismatch and leakage currents. Also charge pump 


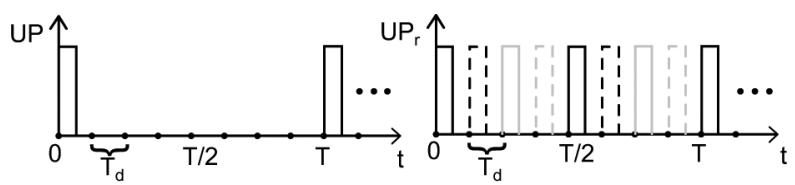

(a)

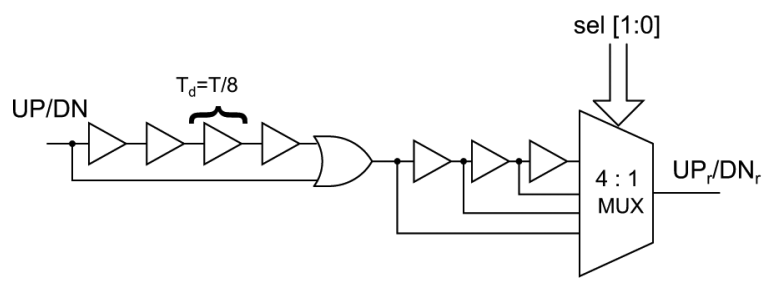

(b)

Fig. 8. (a) The UP pulse before and after applying the technique. (b) Implementation details of combined 4-PPM+2-PR technique.

switch sizes and mismatch may not scale with the charge pump currents, leading to an increase in the net error current injected into the loop filter due to nonidealities. In that case as both PR and PPM offer the same spur rejection, the PR technique can perform poorly when compared to PPM in the presence of delay variations, since the magnitude of the inherent spur is larger.

A combination of PR and PPM results in a better performance. To achieve the same spur rejection of 8-PPM and 8-PR, we apply 4-PPM in conjunction with 2-PR, by passing the pulse through a 2-tap moving average filter $C_{2}(f)$ given by $C_{2}(f)=$ $\left(1+e^{-j \pi f T}\right) / 2$ and then randomizing the filtered pulse positions to four values spaced $T / 8$ seconds apart (this ensures that all the 8 pulse positions are occupied). Fig. 8(a) shows the UP/DN pulses before randomization and the possible positions occupied after applying the technique. The spectrum of the resulting signal is ${ }^{2}$

$\frac{1}{T^{2}} \sum_{k} \delta\left(f-k f_{r}\right)|C(f)|^{2}\left|C_{2}(f)\right|^{2}+\frac{1}{T}\left(1-|C(f)|^{2}\right)\left|C_{2}(f)\right|^{2}$

where $C(f)$ is the pseudofilter associated with the 4-PPM, $C(f)=0.25 \sum_{k=0}^{3} e^{-j \pi f k T / 4} \cdot C_{2}(f)$ has zeroes at odd harmonics of $f_{r}$ and $C(f)$ has zeroes at even harmonics of $f_{r}$ except at multiples of $8 f_{r}$. We can easily verify that

$$
C(f) \cdot C_{2}(f)=\frac{1}{8} \sum_{k=0}^{7} e^{-j \pi f k T / 4}=C_{8}(f)
$$

which is equivalent to an 8-tap moving average filter. The spectrum of the signal reduces to

$$
\frac{1}{T^{2}} \sum_{k} \delta\left(f-8 k f_{r}\right)+\frac{1}{T}\left(1-|C(f)|^{2}\right)\left|C_{2}(f)\right|^{2} .
$$

Equation (15) shows that the spur rejection equals that of 8-PPM and 8-PR. The redistributed noise is also lower compared to 8-PPM as it gets filtered by $C_{2}(f)$. One can intuitively see that the current pulse is passed through a 2-tap moving average filter (2-PR), which eliminates the odd harmonics of $f_{r}$ and when

\footnotetext{
${ }^{2}$ The spectrum can be obtained by first deriving the spectrum of the signal for 4-PPM with $T_{d}=T / 8$ using (10) and then multiplying the resulting spectrum by $\left|C_{2}(f)\right|^{2}$ (since 2-PR is equivalent to passing the signal through the filter $C_{2}(f)$ ).
}

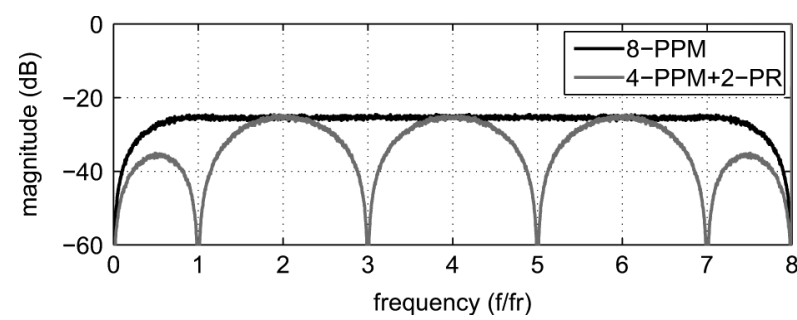

Fig. 9. The spectrum of the 8-PPM signal overlaid with the 4-PPM+2-PR signal (PSD computed with a resolution binwidth of $f_{r} / 512$ ).

4-PPM is applied, the power concentrated in the remaining even harmonics (other than harmonics of $8 f_{r}$ ) are redistributed as noise. Since the total power in the reference harmonics is reduced after 2-PR, the redistributed noise in the 4-PPM+2-PR is smaller compared to 8-PPM.

Fig. 9 shows the simulated spectrum of the 4-PPM+2-PR signal overlaid with the spectrum of the 8-PPM signal. As expected, the noise with the combined technique is lower compared to 8-PPM. Fig. 8(b) shows the implementation details of the 4-PPM+2-PR technique. The repeated UP/DN pulse is generated by first passing the pulse and a half cycle delayed version of the pulse to a two input OR gate and then pulse positions of the repeated pulse are randomly selected using a 4:1 MUX based on a two bit control word sel[ $[1: 0]$. The implementation complexity is also reduced compared to 8-PPM as the complexity of multiplexer and the logic generating the select signals is now reduced.

\section{Shaped $P P M+P R$}

When the magnitude of the spur is high, the redistributed noise is correspondingly high. The noise is then passed through the PLL transfer function which provides high gain for 'midband' frequencies $\left(f_{r} / 100\right.$ to $\left.f_{r} / 10\right)$ inside the bandwidth of the PLL. In cases where the PLL has very low phase noise requirements the redistributed noise may form a lower bound on the noise floor in this region. To resolve this problem the order of noise shaping ${ }^{3}$ can be increased.

The PSD of the PPM signal when modulated by an i.i.d. sequence $a_{i}$, depends only on the probability distribution of $a_{i}$. When the samples of $a_{i}$ are correlated, the resulting spectrum not only depends on its probability distribution but also on its correlation properties. The correlation properties of $a_{i}$ can be exploited to control the shape of the redistributed noise. The spectrum of PPM becomes too complex to compute analytically when $a_{i}$ is correlated for the general case (for any $N$ ). Fortunately it is very easily tractable for $N=2$. The spectrum $S_{r c}(f)$ of the shaped PPM (SPPM) signal when $a_{i}$ takes on binary values ( 0 and 1$)$ with equal probability $(p=0.5)$, is given by (Appendix I)

$$
S_{r c}(f)=\frac{1}{T^{2}}\left|C_{2}(f)\right|^{2} \sum_{k} \delta\left(f-k f_{r}\right)+\frac{4 \sin ^{2}\left(\frac{\pi f T}{2}\right)}{T} S_{a}(f)
$$

where $S_{a}(f)$ is the PSD of $a_{k}$ and $C_{2}(f)$ is the associated 2-tap pseudo moving average filter. Equation (16) shows that the redistributed noise depends upon the PSD $S_{a}(f)$ of $a_{i}$. Thus, the

\footnotetext{
${ }^{3} \mathrm{~A}$ similar approach albeit for a different purpose is proposed in [10].
} 
redistributed noise can be tailored to shape the noise further to higher frequencies by controlling the PSD of $a_{i}$. If the sequence $a_{i}$ has a high-pass spectrum, from (16) the order of the high-pass shaped redistributed noise is $G+1$, where $G$ is the high-pass order of $S_{a}(f)$.

One can realize an $N$-SPPM using an $m$-bit shaped sequence $\left(N=2^{m}\right)$ generated using $m$ independent one bit shaped sequences $^{4}$.

The shaped PPM will increase the high-frequency noise floor and hence it has to be used in conjunction with PR technique to reduce high-frequency noise. For $N=8$, the two possible combinations are one bit SPPM with 4-PR (2-SPPM+4-PR) and two bit SPPM with 2-PR (4-SPPM+2-PR). Though using a 4-PR technique results in a lower high-frequency noise, it suffers from the aforementioned problems of gain saturation and net increase in spur magnitude in the presence of nonidealities like 8-PR. Hence we go for 4-SPPM+2-PR technique. A 4-SPPM+2-PR technique is the same as 4-PPM+2-PR except that the modulating sequence is a two bit higher order shaped sequence. In the current work we chose a third-order shaped sequence to achieve low midband noise. The two bit shaped sequence was generated by combining two independent third-order shaped one bit sequences (generated as described in later sections). The PSD $S_{a}(f)$ of the two bit sequence is

$$
S_{a}(f)=5|\sin (\pi f T) \sin (2 \pi f T) \sin (4 \pi f T)|^{2} .
$$

The pseudofilter and the 2-tap moving average filter associated with this technique are similar to those in the 4-PPM+2-PR technique. This ensures that the reference spurs up to $8 f_{r}$ are absent. The redistributed noise however depends on the PSD $S_{a}(f)$ of two bit sequence $a_{i}$ and the Fourier transform of its squared autocorrelation $\left(R_{a}(i)\right)^{2}$ (we have not presented the results due to the complexity of the expressions involved). Thus, the noise is not truly third-order shaped due to the additional component in the redistributed noise. Nevertheless it leads to a significant reduction in the noise in the midband frequency range. Fig. 10 shows the spectrum of the 4-SPPM+2-PR overlaid with 8-PPM. The high-frequency noise is lower than the 8-PPM technique for most of the frequencies and peaks at some points which is expected in a shaped PPM. The midband noise is orders of magnitude less due to aggressive noise shaping of the SPPM technique.

The implementation of the 4-SPPM+2-PR is similar to the 4-PPM+2-PR technique as shown in Fig. 8, where the two bit control signal is the shaped two bit random sequence.

\section{Delaying NARROW UP/DN PULSES}

To obtain a delay of $T_{d}$, we need an infinite bandwidth system with transfer function $e^{-s T_{d}}$. An inverter is a delaying system with a finite bandwidth. This restricts the minimum pulse width that a chain of inverters can delay reliably without attenuating it below the switching threshold. A maximum bandwidth digital delay line can be realised using a long chain of minimum length inverters. In a $0.18 \mu \mathrm{m}$ CMOS process with a $1.8 \mathrm{~V}$

\footnotetext{
${ }^{4}$ Extending the results presented in Appendix I, we can readily show that the redistributed noise depends up to $m^{\text {th }}$ power of the autocorrelation function for $m$-bit case. This results in the noise not truly being $(G+1)^{\text {th }}$ order shaped due to the additional terms in the redistributed noise.
}

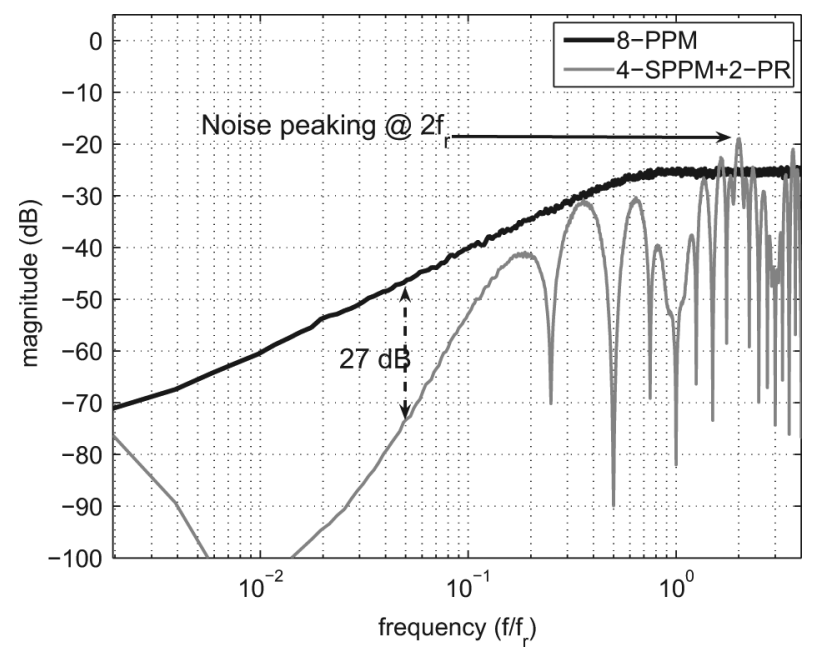

Fig. 10. The spectrum of the 8-PPM signal overlaid with noise shaped 4-SPPM+2-PR signal shown on a log scale for noise comparisons (PSD computed with a resolution binwidth of $f_{r} / 512$ ).

supply to realize a maximum bandwidth delay line of delay 6.25 ns $\left(T_{d}\right)$, the number of minimum length inverters necessary is close to 100 and the average current consumption is $31 \mu \mathrm{A}$. At the slowest process and temperature corners this chain can pass pulses of width $>200 \mathrm{ps}$ without significant attenuation. This minimum delayable pulse width (MPW) reduces as technology scales down.

In a practical charge pump PLL, to improve the linearity of the PFD/CP and thus avoid the problem of dead zone, a nonzero reset delay of $T_{\text {rst }}$ seconds is introduced in the PFD reset path depending upon the size of the charge pump switches and tolerable spur level. This ensures that the UP/DN pulses are "on" for at-least $T_{\text {rst }}$ seconds even when the PLL is in lock condition. The value of $T_{\text {rst }}$ can be close to a few hundred picoseconds ([3], [11]), well above the minimum delayable pulse width. Based on the value of $T_{\text {rst }}$, the delay line is designed by varying the length and supply voltage of the inverters used in the delay chain to obtain the desired delay. In cases where the desired $T_{\text {rst }}$ is close to the minimum delayable pulse width, the delay line needs to have a very high bandwidth. Hence a large number of minimum sized inverters are necessary to realize the desired delay. A straightforward approach to reduce the delay line bandwidth (hence its area and power) is to increase the reset delay more than the desired value (making the UP/DN pulses wider). But it leads to a proportional increase in spur level when fed to the charge pump in the presence of charge pump nonidealities [3]. To resolve this problem, the width of the UP/DN pulses is first increased by $T_{x}$ before feeding it to the delay line and then reduced by the same amount once it is out of the randomizing blocks, before being fed to the charge pump.

Increasing the UP/DN pulse width by $T_{x}$ can be accomplished by increasing the PFD reset delay to $T_{\mathrm{rst}}+T_{x}$ and decreasing the pulse width at the output of the randomizing block can be accomplished using a two input AND gate and a delay $T_{x}$ as shown in Fig. 11. Thus, the minimum UP/DN pulse width seen by the delay line is $T_{\mathrm{rst}}+T_{x}$ and the reset delay pulse width seen by the charge pump is $T_{\text {rst }}$. Even if $T_{\text {rst }}$ is small, $T_{x}$ can be adjusted such that $T_{\text {rst }}+T_{x}$ is wide enough for it to be transmitted through the delay line without 


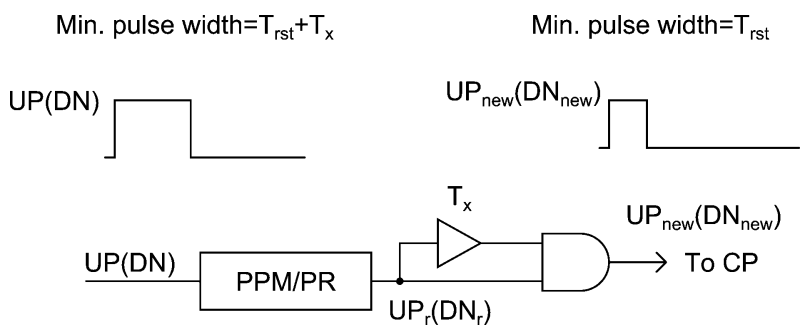

Fig. 11. Implementation of the technique to tackle the narrow pulse problem.

increasing its area and power. For a $T_{\mathrm{rst}}$ of $500 \mathrm{ps}$, to realize a delay of $6.25 \mathrm{~ns}$ in a $0.18 \mu \mathrm{m}$ technology, the number of inverters necessary is close to 60 , with their length equal to $0.25 \mu \mathrm{m}$ and an average current consumption of $18 \mu \mathrm{A}$. Using the aforementioned technique, with $T_{x}=2.5 \mathrm{~ns}$, the number of inverters necessary to realize the same delay across process and temperature variations is 6 , with their length equal to $1.25 \mu \mathrm{m}$ and an average current consumption of $4.3 \mu \mathrm{A}$. Hence the implementation of the delay cell is made simpler and independent of $T_{\text {rst }}$. The implementation techniques presented in Section IV can be used with this minor addition of an AND gate and delay $T_{x}$. In case of PR, the distance between two pulses is $T_{d}$ seconds and hence the delayed pulse should not overlap with the next pulse. This restricts $T_{x}$ to be $<T_{d} / 2$.

Another approach to implement the PPM/PR techniques is to place the delay line before the PFD [4], where the delay lines are driven by the ref and div signals instead of the UP/DN signals. Circuits for PPM, PR, and PPM+PR with this modified architecture are shown in Fig. 12. In this method the width of the pulses is not a concern as the ref/div signals are much wider than the UP/DN signals and the bandwidth of the delay line can be lower (two inverters with their length equal to $3.3 \mu \mathrm{m}$ is sufficient to realize a delay of $6.25 \mathrm{~ns}$ ). Though placing the delay line before PFD might seem attractive due to their small area and power dissipation, a detailed comparison between the two methods (discussed in Section VIII) shows that the delay line after PFD has advantages like better delay mismatch insensitivity, reduced implementation complexity, and relatively smaller delay line area (and current consumption) for the same jitter specification, compared to the former method.

\section{Modulating SeQuence Generation}

In practice the modulating sequence $a_{i}$ is generated using a pseudorandom bit sequence (PRBS) generator. Sequences produced by the PRBS generator are deterministic and periodic [12]. Let $a_{k}$ be a uniformly distributed periodic sequence with a period $M$. Since the modulating signal is periodic, the PPM signal also exhibits a periodic behaviour with a period $T_{p}=$ $M T$. The PSD $S_{r \text {,per }}(f)$ of the periodic PPM signal is given by (derived in Appendix I)

$$
\begin{aligned}
S_{r, \text { per }}(f)=\frac{1}{T^{2}} \sum_{k} \delta(f & \left.-k N f_{r}\right) \\
& +\frac{\left(1-\left|C_{N}(f)\right|^{2}\right)}{M T^{2}} \sum_{k} \delta\left(f-\frac{k f_{r}}{M}\right) .
\end{aligned}
$$

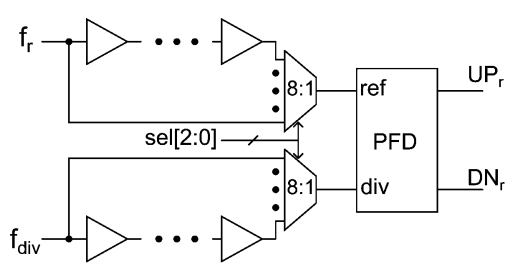

(a)

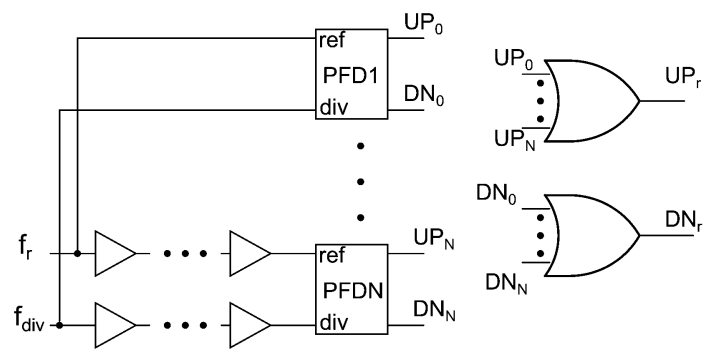

(b)
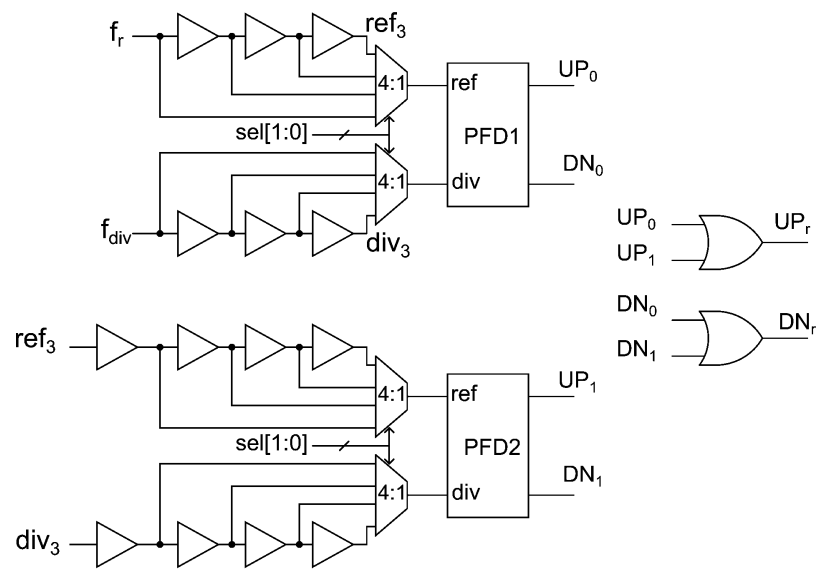

$\mathrm{DN}_{0}$

(c)

Fig. 12. Alternative implementation with delay lines placed before the PFD. (a) PPM. (b) PR. (c) PPM+PR.

The above equation shows that, in a periodic PPM, the noise shaping and the filtering nature of the random PPM are still preserved. But the redistributed noise has only discrete 'frequency slots' (or impulses) over which the noise is spread due to the modulating signal's periodicity (unlike the random case where the noise is spread continuously). This might seem intuitive since the PPM signal is periodic with a period $T_{p}$, its spectrum should have energy concentrated only at the harmonics of $1 / T_{p}$. The harmonics of $f_{r}$ are also the harmonics of $1 / T_{p}=f_{r} / M$, hence the redistributed noise contains spur at $k f_{r}$, but reduced in magnitude. Since $C_{N}\left(k f_{r}\right)=0$ for $k \in[1, N-1]$, for a $\mathrm{N}$-tap moving average filter, we can compute the strength of the reference spurs $k f_{r}$ to be

$$
S_{r, \operatorname{per}}\left(k f_{r}\right)=\frac{1}{M T^{2}} \delta\left(f-k f_{r}\right) .
$$

Comparing the above equation to the spectrum of an unmodulated impulse train, we can see that the strength of the reference harmonics is reduced by a factor $M$. So increasing the periodicity reduces the redistributed noise level (by $3 \mathrm{~dB}$ for twofold increase in $M$ ). One can intuitively see that increasing the time period increases the number of frequency slots over which the 


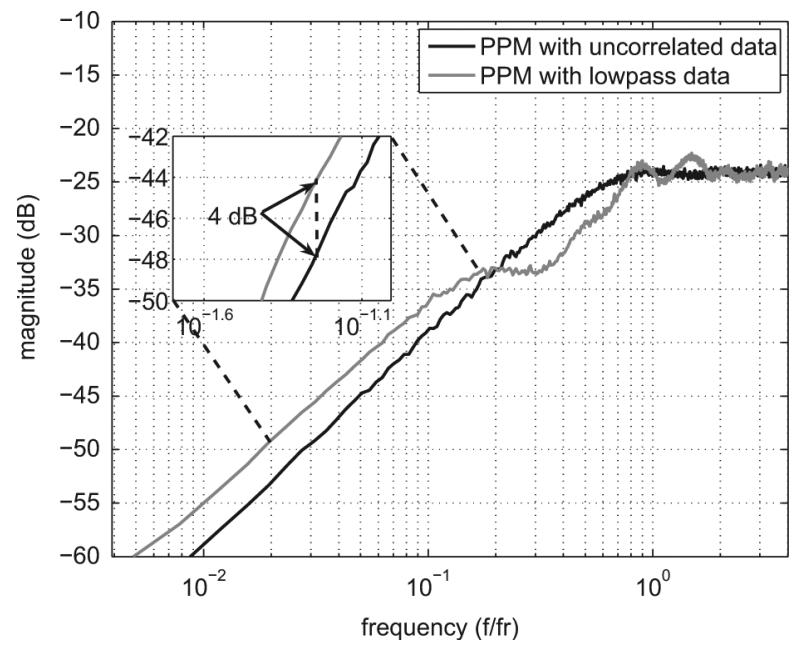

Fig. 13. The spectra of the 8-PPM signal modulated by an uncorrelated (white) data and correlated (low-pass) data shown on log scale for noise comparisons (PSD computed with a resolution binwidth of $f_{r} / 512$ ).

redistributed power can be spread and hence the noise level goes down.

Linear feedback shift registers (LFSR) are used to generate a PRBS. A sequence $a[i]$ constructed using the taps of an LFSR will tend to have a uniform distribution if sufficiently long lengths ${ }^{5}$ are used (as frequency of ones and zeros in the PRBS sequence will approach 0.5). For an LFSR of length $L$, the periodicity of the sequence generated is $M=2^{L}-1$. Increasing $L$ increases the period and hence reduces the noise level. Thus, a long LFSR not only ensures uniform distribution but also a lower redistributed noise floor. For an LFSR of length $15\left(M=2^{15}-1\right)$, the spur is reduced by $10 \log (M)=45.2 \mathrm{~dB}$.

A three bit $(N=8)$ modulating sequence $a[i]$ can be generated using three taps of a single LFSR or by taking each bit from three LFSRs with different feedback configurations. In the latter case the sequence generated will have a "white" spectrum as the samples of the sequence appear uncorrelated to each other. But the implementation complexity is increased as we will need three LFSRs. When the sequence is generated from a single LFSR, the samples of the sequence are correlated as the tap outputs $(z[i])$ of the single LFSR are just shifted versions of each other. For example taking the three consecutive tap outputs of a LFSR, the modulating sequence is given by $a_{c}[i]=\sum_{s=0}^{2} 2^{s} z[i-s]$. The sequence $a_{c}[i]$ possesses a lowpass spectrum. PPM with a low-pass modulating sequence will have higher noise at low frequencies than the case when the modulating sequence is white. Fig. 13 shows the 8-PPM spectrum when modulated by an uncorrelated sequence generated by combining three uncorrelated one bit data, overlaid with the 8-PPM spectrum when modulated by the low-pass sequence $a_{c}[i]$ in the above example. We can see that the spurs are absent in the low-pass case as well, but the low frequency noise floor $\left(f_{r} / 100<f<f_{r} / 10\right)$ increases by $4 \mathrm{~dB}$. Since the increase is marginal, it is not critical as the low frequency noise is dominated by the PFD and charge pump noise. Hence we can generate the three bit sequence from a single LFSR to reduce the implementation complexity.

${ }^{5}$ LFSR length refers to the number of shift registers.

\section{A. Shaped Data Generation}

One method of generating shaped binary data for SPPM is by feeding a uniformly distributed dither at the input of the quantizer of a one bit sigma delta modulator (SDM). This ensures that the output bits generated will have an uniform distribution (equal number of ones and zeroes) but the spectrum is high-pass shaped by the noise transfer function (NTF [13]) of the SDM.

Another way of generating shaped binary random numbers with equal number of zeroes and ones is to use the Manchester encoding given in [14]. Manchester encoding maps a bit 1 to [10] and bit 0 to [01] from a random binary data stream, which provides a first-order shaping [14]. Hence a third-order shaped sequence can be generated by repeating the procedure three times. After the repeated encoding process, bit 1 is mapped to [10010110] and bit 0 is mapped to [01101001]. The magnitude spectrum of the shaped random binary stream (after removing the dc component) can be shown to be $|\sin (\pi f T) \sin (2 \pi f T) \sin (4 \pi f T)|$. This method of generating shaped binary random numbers is very simple to implement because it requires a single LFSR running at $f_{r} / 8$ and a few additional registers. Therefore we choose this method for generating the shaped sequence.

\section{Simulation Results}

To validate the proposed ideas, a PLL was simulated with 1 $\mathrm{MHz}$ bandwidth running at an output frequency of $1 \mathrm{GHz}$ and a reference frequency of $20 \mathrm{MHz}$. The charge pump current ${ }^{6} I_{\mathrm{cp}}$ is $56 \mu \mathrm{A}$ and the loopfilter parameters are $\mathrm{R}=21.7 \mathrm{k} \Omega, \mathrm{C}_{z}=$ $37.25 \mathrm{pF}, \mathrm{C}_{p}=1.99 \mathrm{pF}$. The VCO gain, $K_{\mathrm{vco}}=200 \mathrm{MHz} / \mathrm{V}$, and the nominal divide value is $N_{D}=50$. The PFD, charge pump, and loop filter are at the transistor level and the remaining blocks (VCO, divider and the digital logic for randomization) are modeled behaviorally. ${ }^{7}$ The VCO, divider and the randomization logic are noiseless and the only source of noise is deterministic, contributed by the charge pump and the PFD. To model the effect of charge pump mismatch, a constant current source of value $0.05 I_{\mathrm{cp}}(5 \%$ mismatch $)$ is connected in parallel with the upper current source. The feedthrough is inherent with the circuit of the implemented charge pump. The transistor level schematic of the charge pump is shown in Fig. 14.

The simulated ${ }^{8}$ phase noise of the 8-PPM and 8-PR techniques overlaid with the standard PLL is shown in Fig. 15. The figure shows the absence of reference spurs in both the 8-PPM and 8-PR techniques. The spurs are converted to noise in 8-PPM technique and the noise level is $21.8 \mathrm{~dB}$ below the reference spur (measured at a resolution binwidth of $78.125 \mathrm{kHz}$ ). The 8 -PR technique has only spurs at $8 f_{r}$ without adding any redistributed noise as expected.

The randomization techniques (8-PPM and 4-PPM+2-PR) spread the energy in the harmonics to all the frequencies and

\footnotetext{
${ }^{6}$ The charge pump current is appropriately scaled for the different techniques as explained earlier.

${ }^{7}$ The pulse narrowing circuit is not included in simulations as it does not affect the spur.

${ }^{8}$ The phase noise of the PLL is obtained by computing the PSD of the output VCO phase, which is obtained by running a transient simulation of the PLL and integrating the zero mean control voltage $v_{c}(t)$ after settling, i.e., $\phi_{\text {out }}=$ $2 \pi K_{\mathrm{vco}} \int v_{c}(t) d t$.
} 


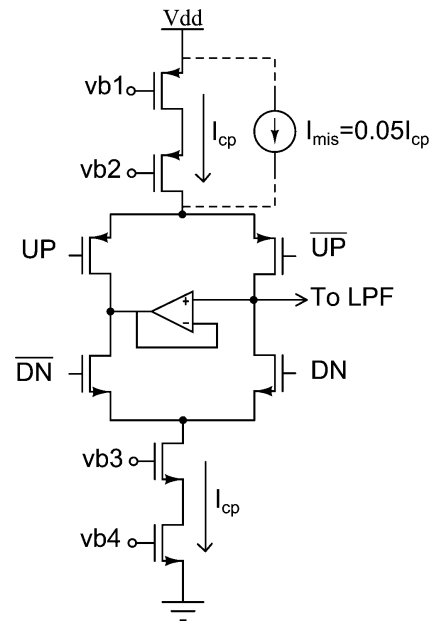

Fig. 14. Transistor level charge pump schematic.

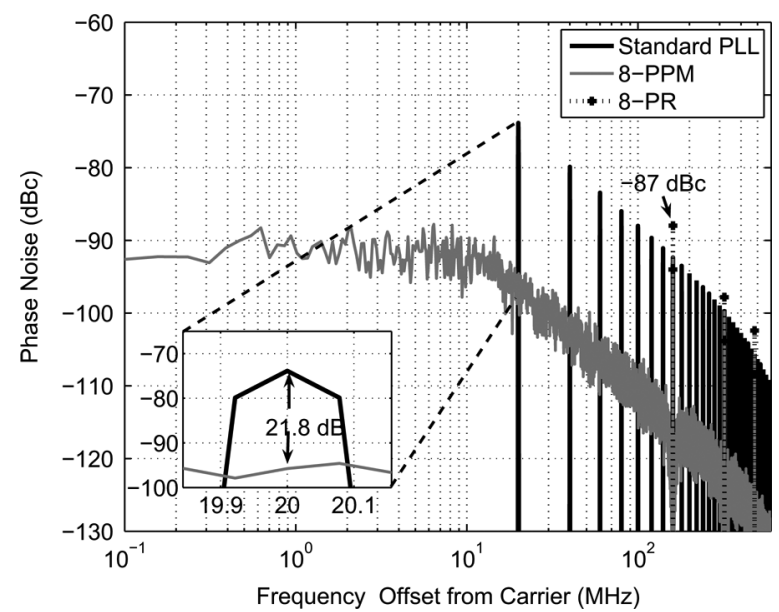

Fig. 15. Phase noise of the PLL output comparing the performance of the 8-PPM and 8-PR techniques with the standard PLL (the resolution bandwidth used for PSD computation is $78.125 \mathrm{kHz}$ )

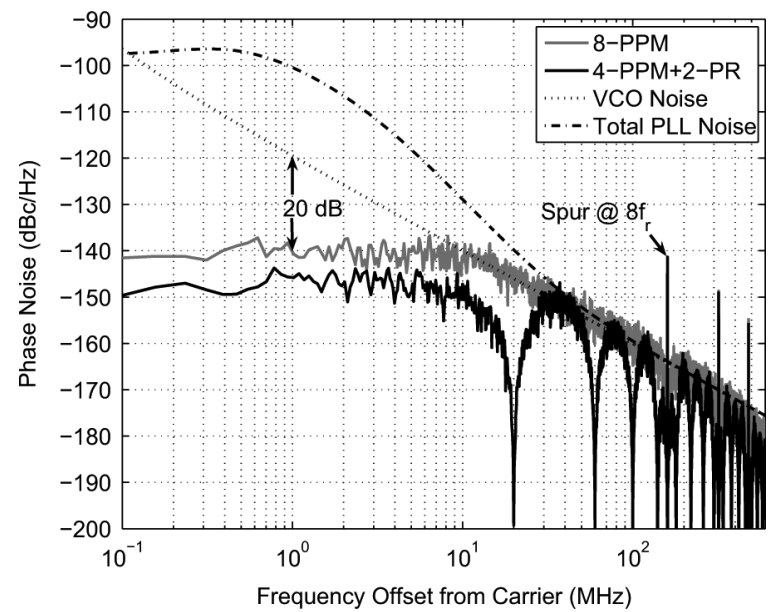

Fig. 16. Phase noise at the PLL output due to the resistor, VCO and charge pump compared with the redistributed noise added by 8-PPM and 4-PPM+2-PR techniques.

hence raise the noise floor at the PLL output. To study the effect of the techniques on the output phase noise we compare the phase noise contribution of the $\mathrm{VCO}$, loop filter resistor and charge pump to the noise introduced by 8 -PPM and 4-PPM+2-PR techniques. Fig. 16 shows the phase noise due to the randomization overlaid with the PLL noise at the output.
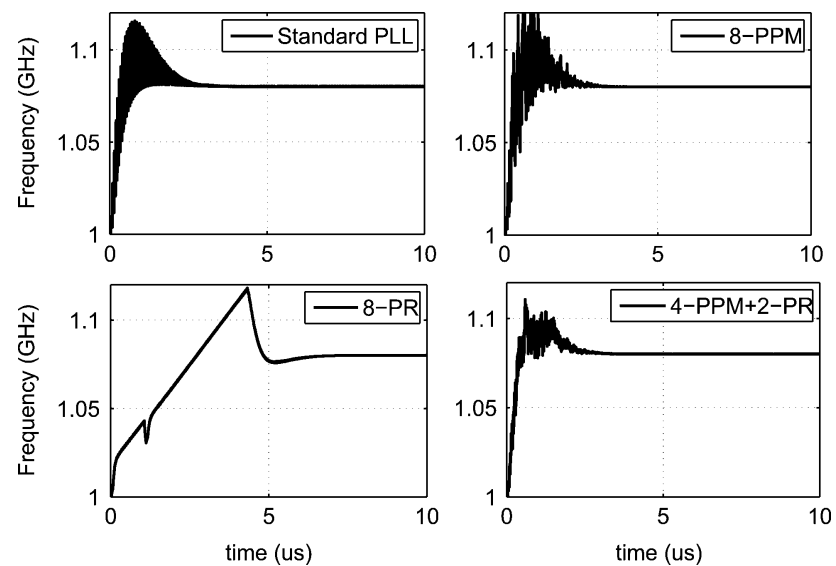

Fig. 17. Settling behavior of the PLL for different techniques for a frequency step of $80 \mathrm{MHz}$ (from $1 \mathrm{GHz}$ to $1.08 \mathrm{GHz}$ ).

The phase noise model of the open loop VCO assumes a phase noise specification of $120 \mathrm{dBc} / \mathrm{Hz}$ at $1 \mathrm{MHz}$ offset and a $1 / f^{3}$ corner of $200 \mathrm{kHz}$ as shown in Fig. 16. The plot shows that the noise added by the randomization techniques is $20 \mathrm{~dB}$ lower than the $\mathrm{VCO}$ noise $^{9}$ at frequencies close to the bandwidth of the PLL and it becomes dominant only at frequencies greater than $f_{r}(20 \mathrm{MHz}$ in the simulated PLL). We can also see from Fig. 16 that the combined 4-PPM+2-PR technique has lower redistributed noise due to the additional filtering offered by 2-PR as explained before.

As mentioned earlier in Section IV-B, the 8-PR technique has the problem of gain saturation for large phase/frequency errors which leads to an increased settling time. So to compare the large-signal settling of the PLL when the different techniques are applied, we give a step of 4 in the divide value (50 to 54) which corresponds to an output frequency step of $4 f_{r}=$ $80 \mathrm{MHz}$ from $1 \mathrm{GHz}$ to $1.08 \mathrm{GHz}$. Fig. 17 shows the simulated response of the PLL to a frequency step when different techniques were applied. The figure shows that the settling behaviour of 8-PPM and 4-PPM+2-PR techniques is similar to that of standard PLL. The PR technique shows a slewing behaviour due to the gain saturation for nearly $4.5 \mu$ s and then settles to the desired value after $8 \mu$ s (approximately twice as much as in the other techniques).

Fig. 18 shows the spectrum of the 4-SPPM+2-PR technique when applied to the implemented PLL and compared with the 8-PPM technique. We can clearly see the shaping of the noise for low frequencies, leading to orders of magnitude reduction in the "midband" region. The simulated PLL shows a reduction in the noise level by $27 \mathrm{~dB}$ near the PLL bandwidth $\left(f_{r} / 20\right)$ compared to the 8-PPM technique.

\section{EFFECTS of DELAY LINE NONIDEALITIES}

The delays are implemented using CMOS inverters and are prone to process variations, random mismatch, and device noise (thermal and flicker). The effect of process variations is discussed in Section III-A. The mismatch in the delay lines leads to a reference spur and the noise in the delay lines increases

${ }^{9}$ Though the noise added is $40 \mathrm{~dB}$ smaller compared to the $\mathrm{CP}$ and resistor noise as shown in the Fig. 16, the noise requirements might be stringent in applications demanding very low in-band noise. To meet those specifications CP and resistor noise can be reduced further. In such cases the noise near the bandwidth will be limited by the VCO noise. 


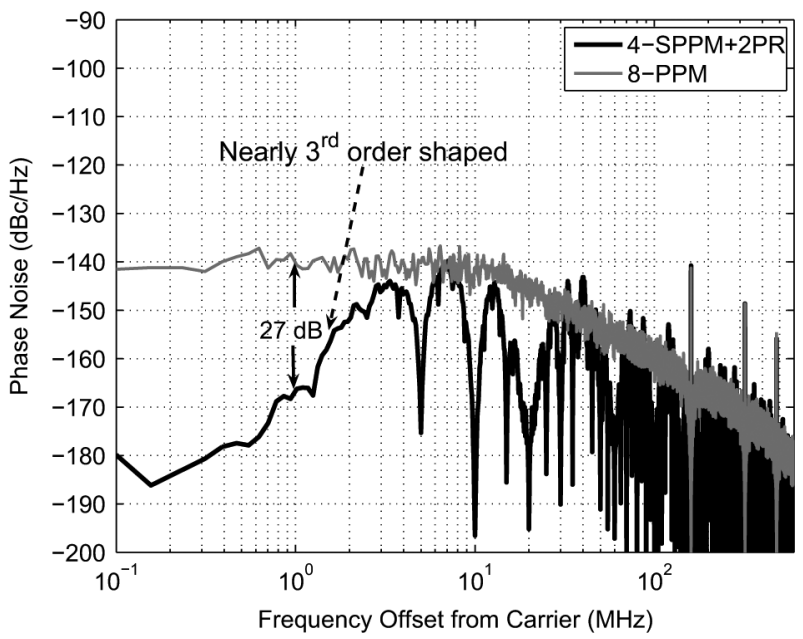

Fig. 18. The phase noise contribution of the 8-PPM overlaid with the phase noise due to 4-SPPM+2-PR technique.

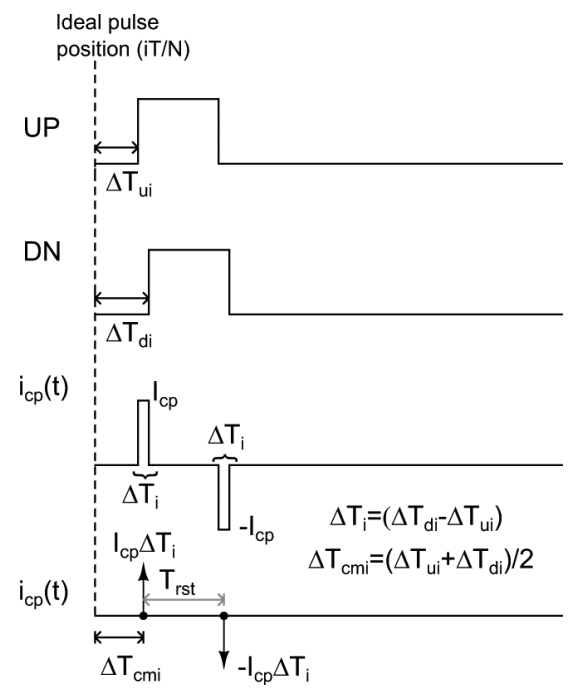

Fig. 19. The UP/DN pulse waveforms and the charge pump current in the presence of delay variations corresponding to a delay of $i T / N$.

the noise floor at the VCO output. The detailed analysis is discussed in the remainder of the section.

\section{A. Effect of Mismatch}

In the presence of mismatch, a delay of $i T / N$ in the UP and DN signal paths, becomes $i T / N+\Delta T_{u i}$ and $i T / N+\Delta T_{d i}$ respectively as shown in Fig. 19. To analyze its effect on the PLL performance, we split the delay variation into common mode and differential components. When these pulses are fed to a charge pump, the common mode component in the delay $i T / N+\left(\Delta T_{u i}+\Delta T_{d i}\right) / 2$ leads to an additional time shift in the current pulse from its ideal position and the differential component in the delay $\Delta T_{d i}-\Delta T_{u i}$ produces a zero average current pulse whose width is equal to $\Delta T_{d i}-\Delta T_{u i}$ as shown in Fig. 19. The narrow charge pump current pulses can be modeled as impulses spaced $T_{\text {rst }}$ seconds apart ${ }^{10}$ with weights given by the area under the pulses and delayed by the common mode component.

\footnotetext{
${ }^{10}$ In the analysis it is assumed that only the delay cell is the source of noise and hence the width of UP/DN pulses is equal to $T_{\text {rst }}$ (the reset delay seen by the charge pump).
}

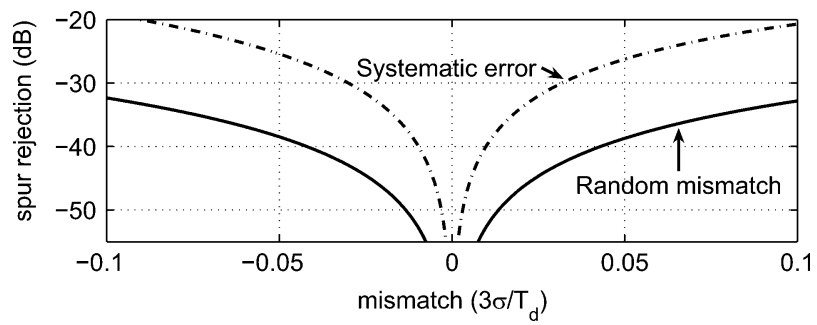

Fig. 20. Spur rejection versus random delay mismatch.

Let $\Delta T$ be variation (due to device mismatch or random device noise) in the delay of single delay cell of value $T / N$ (where $\Delta T$ is a zero mean Gaussian random variable with variance $\sigma_{\Delta T^{2}}{ }^{2}$. A delay of $i T / N$ is obtained by passing the pulse through $i$ identical delay cells of value $T / N$. Assuming that the variations in the delay cells are independent of each other, the variance associated with the delay $i T / N$ is $i \sigma_{\Delta T}{ }^{2}$. Thus, the variance of random variables $\Delta T_{u i}$ and $\Delta T_{d i}$ is $i \sigma_{\Delta T^{2}}$. Let $\Delta T_{c m i}=\left(\Delta T_{u i}+\Delta T_{d i}\right) / 2$ and $\Delta T_{i}=\Delta T_{d i}-\Delta T_{u i}$ be the common mode and differential variations in the delay $i T / N$. Then $\sigma_{\Delta T_{c m i}}=\sqrt{i / 2} \sigma_{\Delta T}$ and $\sigma_{\Delta T_{i}}=\sqrt{2 i} \sigma_{\Delta T}$. In the presence of common mode mismatch, the phase shift $i T / N$ becomes $i T / N+\Delta T_{c m i}$, which leads to a change in the delays of the pseudofilter. When the delays vary, the location of the zeroes of the pseudofilter change $\left(f_{z} \neq k / T\right)$ leading to a degradation in spur rejection. The maximum degradation occurs when there is maximum variation in the delays. Since $\Delta T_{c m i}$ is a gaussian random variable, the maximum variation in in the delay $i T / N$ is considered to be $\approx 3 \sigma_{\Delta T_{c m i}}$. The pseudofilter $C(f)$ for a worst case scenario can be written as

$$
|C(f)|=\frac{1}{N}\left|\sum_{i=0}^{N-1} e^{-j 2 \pi f\left(i T / N+3 \sqrt{i / 2} \sigma_{\Delta T}\right)}\right| .
$$

Fig. 20 shows the degradation in spur rejection in the presence of random mismatch. A point to note is that, in the absence of any delay trimming mechanisms the effect of these random mismatch errors will be dominated by the large systematic errors in the delay caused by temperature and process variations as explained in Section III-A. The degradation due to systematic errors $( \pm 10 \%)$ is overlaid with the random mismatch case in Fig. 20 to show its dominance. The differential mismatch produces a zero average current pulse whose amplitude depends upon the delay value selected. The effect of differential mismatch on the PLL output spectrum can be easily understood in case of PR. When a delay of $i T / N$ is selected, the error pulse injected can be expressed as $\left(I_{\mathrm{cp}} \Delta T_{i} / N\right) p_{d}(t-i T / N)$, where $p_{d}(t)=\delta(t)-\delta\left(t-T_{\mathrm{rst}}\right)$. In PR, $N$ delayed versions of the UP/DN signals appear per reference cycle, hence we have $N$ mismatch current waveforms corresponding to the $N$ delayed versions. So a periodic current is injected into the loop filter every reference cycle in the presence of differential mismatch between the delay lines. Thus, differential mismatch is an additional source of reference spur. The worst case scenario occurs when the mismatches between the delays in the UP/DN delay lines add up in the same polarity and $\Delta T_{i}=3 \sqrt{2 i} \sigma_{\Delta T}$. The 
periodic charge pump current injected per reference cycle due to the differential mismatch can be expressed as

$$
i_{n c p}(t)=\frac{\sqrt{2} I_{\mathrm{cp}} 3 \sigma_{\Delta T}}{N} \sum_{i=0}^{N-1} \sqrt{i} p_{d}\left(t-\frac{i T}{N}\right) .
$$

The magnitude of the spur $(\mathrm{dBc})$ using (1) is given by

$$
20 \log \left|P_{d}\left(f_{r}\right) \frac{Z\left(f_{r}\right) K_{\mathrm{vco}}}{f_{r}} \frac{\sqrt{2} I_{\mathrm{cp}} 3 \sigma_{\Delta T}}{N T} \sum_{i=0}^{N-1} \sqrt{i} e^{-j 2 \pi i f_{r} T / N}\right| .
$$

$P_{d}(f)=1-e^{-j 2 \pi f T_{\mathrm{rst}}}$ is the Fourier transform of the pulse $p_{d}(t)$ which is first-order high-pass shaped. In case of the PPM based techniques, on an average over a large number of reference cycles, all the error waveforms appear equal number of times (since $a_{i}$ is uniformly distributed) within a reference cycle. Hence the average current waveform per reference cycle is the same as in PR and so is the magnitude of the reference spur. Thus, all the techniques produce spurs of the same magnitude. The PPM based techniques additionally add redistributed noise at the PLL output. For a $T_{\text {rst }}$ of 780 ps and a $5 \%$ mismatch in a single delay cell $\left(3 \sigma_{\Delta T}=0.05 T / N\right)$, the reference spur introduced is $-61.6 \mathrm{dBc}$. To remove this component of spur, the UP and DN delay lines can be randomly interchanged based on an additional random signal. The noise added by this randomization will have insignificant contribution at dc due to the high-pass nature of the pulse $p_{d}(t)$. However, it adds to the implementation complexity of the logic. When the delay lines are placed before the PFD, only the positive half of the error current (in Fig. 19) is injected into the loop filter in the presence of mismatch. The PLL responds in a way such that the average current injected into the loop filter every reference cycle is zero. Thus, the per cycle error current waveform in case of PR can be represented as

$$
i_{n c p}(t)=\frac{\sqrt{2} I_{\mathrm{cp}} 3 \sigma_{\Delta T}}{N} \sum_{i=0}^{N-1}(\sqrt{i}-\alpha) \delta\left(t-\frac{i T}{N}\right)
$$

where $\alpha=(1 / N) \sum_{i} \sqrt{i}$. The magnitude of the spur for the same mismatch as before can be computed to be $-41.25 \mathrm{dBc}$. The magnitude of the spur is $20 \mathrm{~dB}$ higher than the former case, where there is a significant reduction in spur due to the high-pass nature of the pulse $p_{d}(t)$. Randomizing the mismatch in this case will directly affect the inband noise as well due to the absence of pulse shaping. This is one major disadvantage of placing the delay line before PFD. Results from behavioral transient simulations show that the spur reduction when the delay lines were placed after PFD was $19.1 \mathrm{~dB}$ more than when they were placed before the PFD (close to the value predicted by analysis).

When the pulse narrowing circuits (Fig. 11) are used in the UP and DN paths, there will be mismatch between $T_{x}$ values used in the two paths. This mismatch appears as a dc phase offset (as every pulse is passed through the circuit) and gets corrected by the PLL. Thus, no additional spur is created due to the mismatch between the delays in the pulse narrowing circuits.
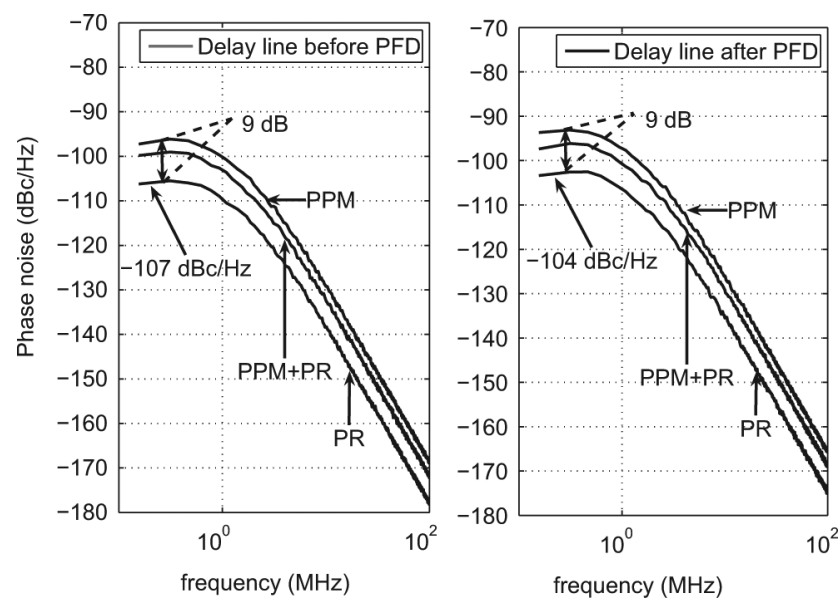

Fig. 21. Phase noise due to delay line noise.

\section{B. Effect of Noise}

In the presence of delay line noise, the rising and falling edges of the UP/DN pulses are corrupted by jitter at the output of the delay line. This leads to an injection of noise current at both these edges spaced $T_{\text {rst }}$ seconds apart into the loop filter. The noise analysis can also be carried in a similar manner by splitting the error as common mode and differential components. Unlike the case of delay mismatch, the variations in the rising and falling edge are not the same due to the uncorrelated nature of the noise. The common mode component of the delay line noise can be treated as a random variation in the delays of the pseudofilter. This leads to degradation in the spur rejection. But this effect will be negligible compared to the effect of mismatch due to its small magnitude and hence it can be ignored.

The differential component of the delay noise however is an additive noise at the charge pump output and hence degrades the output phase noise. The jitter in the rising and falling edges can be treated as uncorrelated and identical noise sources. If $S_{n}(f)$ is the noise current density due to the rising edge jitter, then the total noise current density is $2 S_{n}(f)$ (the sum of two uncorrelated noise PSDs).

When the delay lines are placed before the PFD [4], the jitter of the delay line acts as an input phase error to the PLL and the PFD measures the phase difference between the rising edge of the ref and div signals. So only the error current corresponding to the rising edge is injected into the loop filter and the noise current spectral density is given by $S_{n}(f)$. Thus, the noise added due to delay line jitter is $3 \mathrm{~dB}$ lower compared to the former case.

Fig. 21 shows the PLL output phase noise when the delay line is placed before and after the PFD. For the computations, each delay cell (of delay $6.25 \mathrm{~ns}$ ) was modeled to have an rms jitter of $2.8 \mathrm{ps}^{11}$. The inband noise as shown in the figure is $-107 \mathrm{dBc} / \mathrm{Hz}$ and $-104 \mathrm{dBc} / \mathrm{Hz}$ when the delay lines are placed before and after PFD in case of PR. Thus, with the same delay line jitter before and after PFD, the output phase noise is $3 \mathrm{~dB}$ more for the latter.

\footnotetext{
${ }^{11}$ The jitter was computed using the phase noise analysis in "spectre" on a delay line built using six CMOS inverters in $0.18 \mu \mathrm{m}$ technology with an MPW $>3 \mathrm{~ns}\left(T_{\mathrm{rst}}+T_{x}=3 \mathrm{~ns}\right.$ and $\left.T_{\mathrm{rst}}=780 \mathrm{ps}\right)$ and their supply held at $1.8 \mathrm{~V}$. Also the same delay line is used before and after PFD.
} 
Also the figure shows that the PR technique adds the least noise among all the proposed techniques. In PR the amplitude of each pulse is $1 / N$ of that in PPM, resulting in a $1 / N^{2}$ times noise power per pulse. Since there are $N$ pulses per period in PR as opposed to a single pulse in PPM, the total noise power due to the delay line jitter in PR is $N / N^{2}=1 / N$ of that of PPM ( 9 $\mathrm{dB}$ less for $N=8$ ). Thus, making PR desirable due to the low noise levels associated with it.

When the delay lines are placed before the PFD, fewer inverters of lower bandwidth can be used to realize a given delay compared to when the delay lines are placed after PFD. But to a first order, for a given delay and jitter specification, the power consumed by a short chain of low bandwidth inverters is the same as the long chain of high bandwidth inverters. Also simulations show that the gate area increases gradually as the delay line bandwidth reduces (or as device length increases). Therefore no significant benefit is gained in placing the delay line before PFD, besides the $3 \mathrm{~dB}$ difference in noise mentioned above. This advantage has to be weighed against the significantly large spur due to mismatch between UP/DN delay lines as shown before, and, in case of PR, increased implementation complexity (multiple PFDs). In the authors' opinion, it is preferable to place the delay line after the PFD and use the technique shown in Fig. 11 to increase the pulse width to a value that results in convenient bandwidth and the number of inverters in the delay line.

When the pulse narrowing circuits (Fig. 11) are used in the UP and DN paths, the jitter in the delay $T_{x}$ directly contributes to the inband noise. But since the delay $T_{x}$ is much smaller compared to the large delays of the delay lines in the randomizing blocks, its effect on the output phase noise is negligible compared to that of the latter.

\section{CONCLUSIONS}

PPM of charge pump current pulses by a uniformly distributed sequence behaves as a moving average filter and converts the filtered out harmonics to first-order high-pass shaped wideband noise. The noise added is insignificant at frequencies close to dc, hence it does not affect the long-term jitter of the PLL. Different spur reduction techniques based on PPM and PR were discussed, with their implementation details. Methods of increasing the order of noise shaping in PPM to further reduce the midband noise contributed by random PPM were discussed. The performance degradation of the techniques in the presence of modulating sequence periodicity, random mismatch in the delays and delay line noise are discussed in detail. Design issues in delaying narrow UP/DN pulses is discussed and alternative methods of implementation are presented. The mismatch in the delay line causes a much smaller degradation in spur performance when the delay lines are placed after PFD. Simulation results confirm the correctness of the derived results, reduction in spur levels and low noise levels added by the PPM techniques.

\section{APPENDIX}

The pulse position and amplitude modulated signal $r_{p}(t)$ is given by $r_{p}(t)=\sum_{k} x_{k} p\left(t-k T-a_{k} T_{d}\right)$. It can be expressed as $r_{p}(t)=r(t) * p(t)$ where

$$
r(t)=\sum_{k=-\infty}^{\infty} x_{k} \delta\left(t-k T-a_{k} T_{d}\right)
$$

$r(t)$ is an impulse train whose amplitude is modulated by the sequence $x_{k}$ and the impulse positions are modulated by $a_{k}$. Let $x_{k}$ and $a_{k}$ be stationary sequences ${ }^{12}$ such that $a_{k}$ is an integer valued sequence $\in[0, N-1]$. Let $R_{x}(k)$ be the autocorrelation function of $x_{k}$ and $S_{x}(f)$ its PSD given by $S_{x}(f)=$ $\sum_{k} R_{x}(k) e^{-j 2 \pi f k T}$. For a cyclostationary random process of period $T$, the autocorrelation can be computed as a time average of the ensemble autocorrelation function $\left(R_{r}(t, \tau)\right)$

$$
R_{r}(\tau)=\lim _{L \rightarrow \infty} \frac{1}{L T} \int_{-L T / 2}^{L T / 2} R_{r}(t, \tau) d t
$$

where $R_{r}(t, \tau)=E[r(t) r(t-\tau)]$. Hence

$R_{r}(t, \tau)$

$=E\left[\sum_{i} \sum_{j} x_{i} x_{j} \delta\left(t-i T-a_{i} T_{d}\right) \delta\left(t-\tau-j T-a_{j} T_{d}\right)\right]$.

Since $x_{k}$ and $a_{k}$ are independent, and $f(t) \delta\left(t-t_{0}\right)=$ $f\left(t_{0}\right) \delta\left(t-t_{0}\right)$, and $E\left[x_{i} x_{j}\right]=R_{x}(i-j)$. Letting $i-j=k$, we get

$$
\begin{aligned}
R_{r}(t, \tau)= & \sum_{k} \sum_{i} R_{x}(k) \\
& \times E\left[\delta\left(t-i T-a_{i} T_{d}\right) \delta\left(\tau-k T-\left(a_{i}-a_{i-k}\right) T_{d}\right)\right] .
\end{aligned}
$$

It can be easily verified that $R_{r}(t, \tau)$ is also periodic in $t$, with a period $T$. Hence $r(t)$ is a cyclostationary random process. Using (21) we get

$$
\begin{aligned}
& R_{r}(\tau)=\sum_{k} R_{x}(k) \lim _{L \longrightarrow \infty} \frac{1}{L T} \sum_{i=-L / 2}^{L / 2} \\
& \int_{i T}^{i T+T} E\left[\delta\left(t-i T-a_{i} T_{d}\right) \delta\left(\tau-k T-\left(a_{i}-a_{i-k}\right) T_{d}\right)\right] d t
\end{aligned}
$$

we define $b_{k}=a_{i}-a_{i-k}$, as a new sequence. Since the sequence $a_{k}$ is stationary, the statistics of $b_{k}$ are independent of time origin $i$ and depends only on time difference $k$. Taking the integration inside the expectation operator and after some manipulations we obtain

$$
R_{r}(\tau)=\frac{1}{T} \sum_{k} R_{x}(k) E\left[\delta\left(\tau-k T-b_{k} T_{d}\right)\right] .
$$

Let $P_{A}(a)$ be the probability mass function of the random variable $a$, and $P_{B k}(b)$ be the probability mass function of the random variable $b_{k}$. Then $E\left[\delta\left(\tau-k T-b_{k} T_{d}\right)\right]$

$$
=\sum_{b \in B} P_{B k}(b)\left[\delta\left(\tau-k T-b T_{d}\right)\right]=P_{B k}\left(\frac{\tau-k T}{T_{d}}\right) .
$$

Using the above result in (23) we have

$$
R_{r}(\tau)=\frac{1}{T} \sum_{k} R_{x}(k) P_{B k}\left(\frac{\tau-k T}{T_{d}}\right)
$$

${ }^{12}$ Though we have assumed $x_{k}$ and $a_{k}$ to be stationary, wide sense stationarity [9] is sufficient for the derivations given in this work. 
$P_{B k}\left(\tau-k T / T_{d}\right)$ is a set of impulses spaced $T_{d}$ apart centered on $k T$. The amplitude of the impulses is the probability mass function ${ }^{13}$ of $b_{k}$. Equation (24) can be rewritten as

$$
R_{r}(\tau)=\frac{1}{T} \sum_{k} R_{x}(k) \delta(\tau-k T) * P_{B k}\left(\frac{\tau}{T_{d}}\right) .
$$

Then the spectrum of the PPAM signal is obtained by taking the Fourier transform of (25). To compute the autocorrelation function we need to compute $P_{B k}(b)$ for all $k$. In the reminder of this section, the expressions of the spectrum is derived for different cases of the modulating sequence by computing $P_{B k}(b)$ for all these cases.

PPM by an I.I.D. Sequence: If the sample values of the sequence $a_{k}$ are i.i.d., then $a_{i}$ and $a_{i-k}$ are independent. The probability mass function $P_{B k}(b)$ of $b_{k}$ is the same for all nonzero values of $k$ and is given by the convolution of the $P_{A}(a)$ and $P_{A}(-a)[9]$

$$
P_{B k}(b)= \begin{cases}P_{A}(a) * P_{A}(-a)=P_{B}(b) & \text { for } k \neq 0 \\ \delta(b) & \text { for } k=0\end{cases}
$$

where $P_{B}(b)$ is the time independent probability distribution of the sequence $b_{k}$. Using the above conditions we obtain the expression for $R_{r}(\tau)$ from (25) to be

$$
\begin{aligned}
\left(\frac{1}{T} \sum_{k} R_{x}(k) \delta(\tau-k T)\right) * & P_{B}\left(\frac{\tau}{T_{d}}\right) \\
& +\frac{R_{x}(0)}{T}\left[\delta(\tau)-P_{B}\left(\frac{\tau}{T_{d}}\right)\right] .
\end{aligned}
$$

The PSD $S_{r}(f)$ of the signal $r(t)$ is obtained by taking the Fourier transform of the above result. Since $P_{A}\left(\tau / T_{d}\right)$ is real. If $C(f)$ is the Fourier transform of $P_{A}\left(\tau / T_{d}\right)$, then the Fourier transform of $P_{A}\left(-\tau / T_{d}\right)$ is $C^{*}(f)$. Using (26) the Fourier transform $C_{b}(f)$ of $P_{B}\left(\tau / T_{d}\right)$ is given by

$$
C_{b}(f)=C(f) \cdot C^{*}(f)=|C(f)|^{2} .
$$

Using these results we obtain the spectrum of PPAM signal $r(t)$ modulated by an i.i.d. sequence as

$$
S_{r}(f)=\frac{1}{T} S_{x}(f)|C(f)|^{2}+\frac{R_{x}(0)}{T}\left(1-|C(f)|^{2}\right)
$$

for any arbitrary pulse shape $r_{p}(t)=r(t) * p(t)$. The power spectral density $S_{r p}(f)$ of the PPAM signal $r_{p}(t)$ is given by $S_{r p}(f)=S_{r}(f)|P(f)|^{2}$, where $P(f)$ is the Fourier transform of the pulse $p(t)$. The spectrum $S_{x p}(f)$ of the PAM signal $x_{p}(t)$ is well known [8] and given by $S_{x p}(f)=|P(f)|^{2} S_{x}(f) / T$. Using this we can reduce the spectrum of PPAM to

$$
S_{r p}(f)=S_{x p}(f)|C(f)|^{2}+\frac{R_{x}(0)}{T}\left(1-|C(f)|^{2}\right)|P(f)|^{2} .
$$

One can readily show that for a deterministic sequence $x_{k}$, we obtain the same results as derived above. An important point to

\footnotetext{
${ }^{13}$ If $a_{i}$ is a continuous random variable, $P_{B k}\left(\tau-k T / T_{d}\right)$ is the pdf of $b_{k}$ with a mean $k T$.
}

observe is that the spectrum of a PAM signal is completely determined by the autocorrelation of the samples of the amplitude modulating sequence alone, but in a PPAM signal, the spectrum depends on the probability distribution of the modulating signal $a_{k}$ as well.

PPM by a Pseudorandom Sequence: In practice the modulating sequence $a_{k}$ is generated using a PRBS generator. PRBS sequences are pseudorandom and the sequence repeats itself with a period $M, a_{i}=a_{i+M}$. The sequence $b_{k}=a_{i}-a_{i+k}$ is also periodic with a period $M$ and $b_{k}=0$ for $k=\cdots$ $M, 0, M, 2 M \cdots$. For all other values of $k$, the sequence is the same as the uncorrelated case

$$
\begin{aligned}
& P_{B k}(b) \\
& \quad= \begin{cases}P_{A}(a) * P_{A}(-a)=P_{B}(b) & \text { for }|k| \neq 0, M, 2 M \cdots \\
\delta(b) & \text { for }|k|=0, M, 2 M \cdots\end{cases}
\end{aligned}
$$

Using these constraints in (25) and taking its Fourier transform we obtain the spectrum $S_{r, \text { per }}(f)$ of the PPAM signal modulated by a pseudorandom sequence with period $M$ as

$$
S_{r, \operatorname{per}}(f)=\frac{1}{T} S_{x}(f)|C(f)|^{2}+\frac{\left(1-|C(f)|^{2}\right)}{T} S_{x}\left(\frac{f}{M}\right)
$$

where $S_{x}(f / M)=\sum_{k} R_{x}(k M) e^{-j 2 \pi f k M T}$. When the impulse amplitudes are not modulated $R_{x}(k)=1$, the spectrum reduces to

$\frac{1}{T^{2}}|C(f)|^{2} \sum_{k} \delta\left(f-k f_{r}\right)+\frac{\left(1-|C(f)|^{2}\right)}{M T^{2}} \sum_{k} \delta\left(f-\frac{k f_{r}}{M}\right)$.

PPM by a Binary Correlated Sequence: When the samples of $a_{k}$ are not i.i.d. $P_{B k}(b)$ is not constant and the spectrum of the PPAM signal cannot be expressed in terms of the probability distribution of sequence $a_{k}$ alone. We need to compute $P_{B k}(b)$ for all $k$, which depends on the joint statistics of the sequence $a_{i}$ and $a_{i+k}$. This is very complicated in the general case. However when $a_{k}$ takes on two values the spectrum of PPM is easily tractable.

Let $A$ be a binary discrete random variable with $P_{A}(a=1)=$ $p$ and $P_{A}(a=0)=1-p$. When the sequence $a_{k}$ is correlated

$$
R_{a}(k)=E\left[a_{i} a_{i+k}\right]-\mu^{2} \neq 0 \text { for } k \neq 0
$$

where $\mu=E[A]=p$ is the mean and $\mu^{2}$ is subtracted from $E\left[a_{i} a_{i+k}\right]$ to remove the dc offset. Since the random variable $A$ can take only two values ( 0 and 1 ), the sequence $b_{k}$ takes three values $-1,0$ and 1 with probability $P_{B k}(-1), P_{B k}(0)$ and $P_{B k}(1)$ respectively. To compute the probability distribution of $b_{k}$ we need three equations, since we have three unknowns.

We use the moments of the random variable $b_{k}$ to get the three equations

$$
\begin{aligned}
E\left[b_{k}{ }^{0}\right] & =P_{B k}(-1)+P_{B k}(0)+P_{B k}(1)=1 \\
E\left[b_{k}{ }^{1}\right] & =P_{B k}(-1)-P_{B k}(1)=0 \\
E\left[b_{k}{ }^{2}\right] & =1^{2} . P_{B k}(1)+(-1)^{2} \cdot P_{B k}(-1) \\
& =2\left(R_{a}(0)-R_{a}(k)-p^{2}\right) .
\end{aligned}
$$


Solving the above equations, we get

$$
P_{B k}(b)= \begin{cases}p^{2}+(1-p)^{2}+2 R_{a}(k) & \text { for } b=0 \\ p(1-p)-R_{a}(k) & \text { for } b= \pm 1 \\ 0 & \text { otherwise. }\end{cases}
$$

Thus, the probability distribution $P_{B k}(b)$ of the sequence $b_{k}$ is completely determined by the probability distribution of the sequence $a_{i}$ and its autocorrelation function $R_{a}(k)$ when the random variable $a$ takes only two values. Now that we know $P_{B k}(b)$ for all $k$, we can substitute it in (25) and obtain the spectrum of the PPAM signal $S_{r c}(f)$ modulated by a correlated binary random variable

$$
S_{r c}(f)=\frac{1}{T}|C(f)|^{2} S_{x}(f)+\frac{4 \sin ^{2}\left(\pi f T_{d}\right)}{T}\left(S_{a}(f) * S_{x}(f)\right)
$$

where $S_{a}(f)=\sum_{k} R_{a}(k) e^{-j 2 \pi f k T}$ is the PSD of the sequence $a_{k}, S_{a}(f) * S_{x}(f)$ is the convolution of the two power spectra given by $S_{a}(f) * S_{x}(f)=\sum_{k} R_{a}(k) R_{x}(k) e^{-j 2 \pi f k T}$ and $|C(f)|^{2}=\left|(1-p)+p e^{-j 2 \pi f T_{d}}\right|^{2}$. When the amplitude of the impulses are not modulated $R_{x}(k)=1$, we have

$$
S_{r c}(f)=\frac{1}{T}|C(f)|^{2} \sum_{k} \delta\left(f-k f_{r}\right)+\frac{4 \sin ^{2}\left(\pi f T_{d}\right)}{T} S_{a}(f) .
$$

\section{ACKNOWLEDGMENT}

The authors would like to thank Prof. S. Pamarti for suggestions on generating shaped data [14] and the reviewers whose feedback helped us improve the manuscript.

\section{REFERENCES}

[1] C. S. Vaucher, "An adaptive PLL tuning system architecture combining high spectral purity and fast settling time," IEEE J. Solid-State Circuits, vol. 34, no. 4, pp. 2131-2137, Apr. 2000.

[2] C.-F. Liang et al., "Spur suppression techniques for frequency synthesizers," IEEE Trans. Circuits Syst. II, Exp. Briefs, vol. 54, no. 8, pp. 653-657, Aug. 2007.

[3] C.-F. Liang et al., "A digital calibration technique for charge pumps in phase-locked systems," IEEE J. Solid-State Circuits, vol. 38, no. 2, pp. 390-398, Feb. 2008.

[4] T. C. Lee and W. L. Lee, "A spur suppression technique for phase locked frequency synthesizers," in Proc. IEEE Int. Solid-State Circuits Conf. (ISSCC), Feb. 2006, pp. 592-593.
[5] C. Thambidurai and N. Krishnapura, "Spur reduction in wideband PLLs by random positioning of charge pump current pulses," in Proc. IEEE Int. Symp. Circuits Syst., Jun. 2010, pp. 3397-3400.

[6] A. Maxim et al., "Sample-reset loop filter architecture for process independent and ripple-pole-less low jitter CMOS charge-pump PLLs," in Proc. IEEE Int. Symp. Circuits Syst., May 2001, pp. 766-769.

[7] M. Z. Win, "A unified spectral analysis of generalised time-hopping spread spectrum signals in the presence of timing jitter," IEEE J. Sel. Areas Commun., vol. 38, no. 2, pp. 390-398, Feb. 2008.

[8] B. P. Lathi, Modern Digital and Analog Communication Systems, 3rd ed. New York: Oxford Univ. Press, 1998.

[9] A. Papoulis and S. U. Pillai, Probability, Random Variables and Stochastic Processes, 4th ed. New York: McGraw-Hill, 2002.

[10] B. R. Veillette and G. W. Roberts, "On-chip measurement of the jitter transfer function of charge pump phase-locked loops," IEEE J. SolidState Circuits, vol. 33, no. 3, pp. 483-491, Mar. 1998.

[11] S. Pamarti et al., "A wideband 2.4-GHz delta-sigma fractional-N PLL with 1-Mb/s in-loop modulation," IEEE J. Solid-State Circuits, vol. 39, no. 1, pp. 49-62, Jan. 2004.

[12] Maximum length pseudorandom sequences [Online]. Available: http:// www.newwaveinstruments.com

[13] R. Schreier and G. Temes, Understanding Delta-Sigma Data Converters. Piscataway, NJ: IEEE Press, 2005.

[14] P.-E. Su and S. Pamarti, "Mismatch shaping techniques to linearize charge pump errors in fractional-N PLLs," IEEE Trans. Circuits Syst. I, Reg. Papers, vol. 57, no. 6, pp. 1221-1230, Jun. 2010.

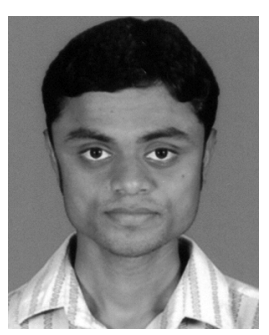

Chembiyan Thambidurai received the B.E. (Hons.) degree from Birla Institute of Technology and Science-Pilani, India. He is currently working toward the M.S. degree at the Indian Institute of Technology-Madras

He is currently a Project Associate at Indian Institute of Technology, Madras. His research interests are wireless transceivers and frequency synthesizers.

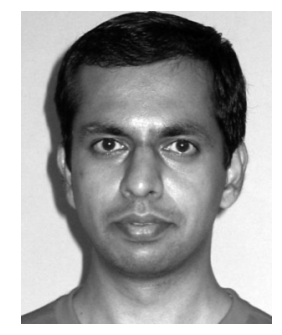

Nagendra Krishnapura received the B.Tech. degree from the Indian Institute of Technology, Madras, and the Ph.D. degree from Columbia University, New York.

He has worked as an Analog Design Engineer at Texas Instruments, Bell Laboratories, Celight Inc., Multilink, and Vitesse semiconductor. He has taught analog circuit design courses at Columbia University as an adjunct faculty. He is currently an Associate Professor at the Indian Institute of Technology, Madras. His interests are analog and RF circuit design and analog signal processing. 